\title{
Study of the $D^{0} p$ amplitude in $\Lambda_{b}^{0} \rightarrow D^{0} p \pi^{-}$decays
}

\section{LHCD}

\section{The LHCb collaboration}

\section{E-mail: Anton.Poluektov@cern.ch}

Abstract: An amplitude analysis of the decay $\Lambda_{b}^{0} \rightarrow D^{0} p \pi^{-}$is performed in the part of the phase space containing resonances in the $D^{0} p$ channel. The study is based on a data sample corresponding to an integrated luminosity of $3.0 \mathrm{fb}^{-1}$ of $p p$ collisions recorded by the LHCb experiment. The spectrum of excited $\Lambda_{c}^{+}$states that decay into $D^{0} p$ is studied. The masses, widths and quantum numbers of the $\Lambda_{c}(2880)^{+}$and $\Lambda_{c}(2940)^{+}$resonances are measured. The constraints on the spin and parity for the $\Lambda_{c}(2940)^{+}$state are obtained for the first time. A near-threshold enhancement in the $D^{0} p$ amplitude is investigated and found to be consistent with a new resonance, denoted the $\Lambda_{c}(2860)^{+}$, of spin $3 / 2$ and positive parity.

KEYwords: B physics, Charm physics, Hadron-Hadron scattering (experiments), QCD, Spectroscopy

ARXIV EPRINT: 1701.07873 


\section{Contents}

1 Introduction 1

2 Detector and simulation 3

3 Amplitude analysis formalism $\quad 4$

3.1 Phase space of the decay $\Lambda_{b}^{0} \rightarrow D^{0} p \pi^{-} \quad 4$

3.2 Helicity formalism 4

3.3 Resonant and nonresonant lineshapes $\quad 7$

3.4 Fitting procedure 8

3.5 Fit parameters and fit fractions 9

3.6 Evaluation of fit quality 9

4 Signal selection $\quad 10$

5 Fit regions and event yields 11

6 Efficiency variation over the Dalitz plot 12

$\begin{array}{lll}7 & \text { Background distribution } & 14\end{array}$

8 Effect of momentum resolution $\quad 14$

9 Amplitude analysis $\quad 15$

9.1 Fit in the nonresonant region $\quad 15$

$\begin{array}{lll}9.2 & \text { Fit in the region of } \Lambda_{c}(2880)^{+} & 15\end{array}$

9.3 Fit in the near-threshold region $\quad 22$

9.4 Fit including $\Lambda_{c}(2940)^{+} \quad 26$

10 Conclusion $\quad 32$

$\begin{array}{ll}\text { The LHCb collaboration } & 38\end{array}$

\section{Introduction}

Decays of beauty baryons to purely hadronic final states provide a wealth of information about the interactions between the fundamental constituents of matter. Studies of direct $C P$ violation in these decays can help constrain the parameters of the Standard Model and New Physics effects in a similar way as in decays of beauty mesons [1-7]. Studies of the decay dynamics of beauty baryons can provide important information on the spectroscopy of charmed baryons, since the known initial state provides strong constraints on the quantum numbers of intermediate resonances. The recent observation of pentaquark states at $\mathrm{LHCb}[8]$ has renewed the interest in baryon spectroscopy. 


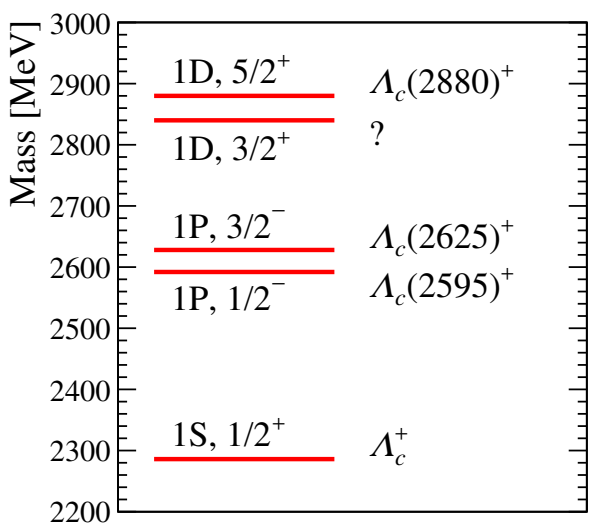

Figure 1. Expected spectrum of the $\Lambda_{c}^{+}$ground state and its orbital excitations from a study based on the nonrelativistic heavy quark-light diquark model [21], along with the observed resonances corresponding to those states [23].

The present analysis concerns the decay amplitude of the Cabibbo-favoured decay $\Lambda_{b}^{0} \rightarrow D^{0} p \pi^{-}$(the inclusion of charge-conjugate processes is implied throughout this paper). A measurement of the branching fraction of this decay with respect to the $\Lambda_{b}^{0} \rightarrow \Lambda_{c}^{+} \pi^{-}$ mode was reported by the LHCb collaboration using a data sample corresponding to $1.0 \mathrm{fb}^{-1}$ of integrated luminosity [9]. The $\Lambda_{b}^{0} \rightarrow D^{0} p \pi^{-}$decay includes resonant contributions in the $D^{0} p$ channel that are associated with intermediate excited $\Lambda_{c}^{+}$states, as well as contributions in the $p \pi^{-}$channel due to excited nucleon $(N)$ states. The study of the $D^{0} p$ part of the amplitude will help to constrain the dynamics of the Cabibbo-suppressed decay $\Lambda_{b}^{0} \rightarrow D^{0} p K^{-}$, which is potentially sensitive to the angle $\gamma$ of the Cabibbo-KobayashiMaskawa quark mixing matrix $[10,11]$. The analysis of the $D^{0} p$ amplitude is interesting in its own right. One of the states decaying to $D^{0} p$, the $\Lambda_{c}(2940)^{+}$, has a possible interpretation as a $D^{*} N$ molecule [12-20]. There are currently no experimental constraints on the quantum numbers of the $\Lambda_{c}(2940)^{+}$state.

The mass spectrum of the predicted and observed orbitally excited $\Lambda_{c}^{+}$states [21] is shown in figure 1 . In addition to the ground state $\Lambda_{c}^{+}$and to the $\Lambda_{c}(2595)^{+}$and $\Lambda_{c}(2625)^{+}$ states, which are identified as the members of the $P$-wave doublet, a $D$-wave doublet with higher mass is predicted. One of the members of this doublet could be the state known as the $\Lambda_{c}(2880)^{+}$, which is measured to have spin and parity $J^{P}=5 / 2^{+}[22,23]$, while no candidate for the other state has been observed yet. Several theoretical studies provide mass predictions for this state and other excited charm baryons [21, 24-29]. The BaBar collaboration has previously reported indications of a structure in the $D^{0} p$ mass spectrum close to threshold, at a mass around $2.84 \mathrm{GeV},{ }^{1}$ which could be the missing member of the $D$-wave doublet [30].

This analysis is based on a data sample corresponding to an integrated luminosity of $3.0 \mathrm{fb}^{-1}$ of $p p$ collisions recorded by the LHCb detector, with $1.0 \mathrm{fb}^{-1}$ collected at centreof-mass energy $\sqrt{s}=7 \mathrm{TeV}$ in 2011 and $2.0 \mathrm{fb}^{-1}$ at $\sqrt{s}=8 \mathrm{TeV}$ in 2012 .

\footnotetext{
${ }^{1}$ Natural units with $\hbar=c=1$ are used throughout.
} 
The paper is organised as follows. Section 2 gives a brief description of the $\mathrm{LHCb}$ experiment and its reconstruction and simulation software. The amplitude analysis formalism and fitting technique is introduced in section 3. The selection of $\Lambda_{b}^{0} \rightarrow D^{0} p \pi^{-}$ candidates is described in section 4 , followed by the measurement of signal and background yields (section 5), evaluation of the efficiency (section 6), determination of the shape of the background distribution (section 7), and discussion of the effects of momentum resolution (section 8). Results of the amplitude fit are presented in section 9 separately for four different regions of the $\Lambda_{b}^{0} \rightarrow D^{0} p \pi^{-}$phase space, along with the systematic uncertainties for those fits. Section 10 gives a summary of the results.

\section{Detector and simulation}

The LHCb detector $[31,32]$ is a single-arm forward spectrometer covering the pseudorapidity range $2<\eta<5$, designed for the study of particles containing $b$ or $c$ quarks. The detector includes a high-precision tracking system consisting of a silicon-strip vertex detector surrounding the $p p$ interaction region, a large-area silicon-strip detector located upstream of a dipole magnet with a bending power of about $4 \mathrm{Tm}$, and three stations of silicon-strip detectors and straw drift tubes placed downstream of the magnet. The tracking system provides a measurement of momentum, $p$, of charged particles with relative uncertainty that varies from $0.5 \%$ at low momentum to $1.0 \%$ at $200 \mathrm{GeV}$. The minimum distance of a track to a primary vertex (PV), the impact parameter (IP), is measured with a resolution of $\left(15+29 / p_{\mathrm{T}}\right) \mu \mathrm{m}$, where $p_{\mathrm{T}}$ is the component of the momentum transverse to the beam, in $\mathrm{GeV}$. Different types of charged hadrons are distinguished using information from two ring-imaging Cherenkov detectors. Photons, electrons and hadrons are identified by a calorimeter system consisting of scintillating-pad and preshower detectors, an electromagnetic calorimeter and a hadronic calorimeter. Muons are identified by a system composed of alternating layers of iron and multiwire proportional chambers.

The online event selection is performed by a trigger [33], which consists of a hardware stage, based on information from the calorimeter and muon systems, followed by a software stage, which applies a full event reconstruction. At the hardware trigger stage, events are required to have a muon with high $p_{\mathrm{T}}$ or a hadron, photon or electron with high transverse energy in the calorimeters. The software trigger requires a two-, three- or fourtrack secondary vertex with significant displacement from any PV in the event. At least one charged particle forming the vertex must exceed a $p_{\mathrm{T}}$ threshold in the range $1.6-1.7 \mathrm{GeV}$ and be inconsistent with originating from a PV. A multivariate algorithm [34] is used for the identification of secondary vertices consistent with the decay of a $b$ hadron.

In the simulation, $p p$ collisions are generated using PyтHIA $8[35,36]$ with a specific LHCb configuration [37]. Decays of hadronic particles are described by EvTGEN [38], in which final-state radiation is generated using Рнотоs [39]. The interaction of the generated particles with the detector, and its response, are implemented using the GEANT4 toolkit [40, 41] as described in ref. [42]. 
(a)

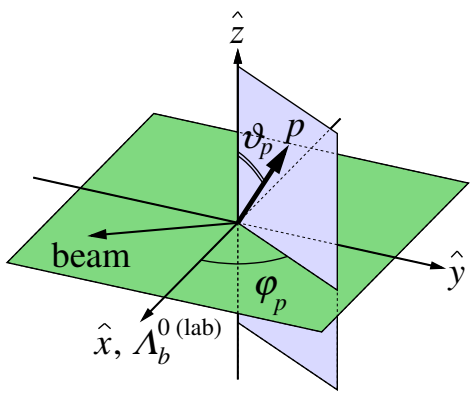

(b)

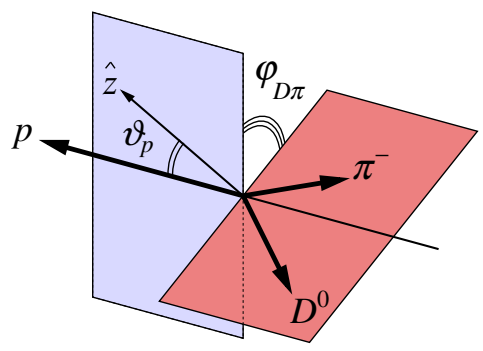

Figure 2. Definition of the angles describing the orientation of the $\Lambda_{b}^{0} \rightarrow D^{0} p \pi^{-}$decay in the reference frame where the $\Lambda_{b}^{0}$ baryon is at rest: (a) $\vartheta_{p}$ and $\varphi_{p}$, and (b) $\varphi_{D \pi}$.

\section{Amplitude analysis formalism}

The amplitude analysis is based on the helicity formalism used in previous LHCb analyses. A detailed description of the formalism can be found in refs. [8, 43, 44]. This section gives details of the implementation specific to the decay $\Lambda_{b}^{0} \rightarrow D^{0} p \pi^{-}$.

\subsection{Phase space of the decay $\Lambda_{b}^{0} \rightarrow D^{0} p \pi^{-}$}

Three-body decays of scalar particles are described by the two-dimensional phase space of independent kinematic parameters, often represented as a Dalitz plot [45]. For baryon decays, in general also the additional angular dependence of the decay products on the polarisation of the decaying particle has to be considered.

A vector of five kinematic variables (denoted $\Omega$ ) describes the phase space of the decay $\Lambda_{b}^{0} \rightarrow D^{0} p \pi^{-}$. The kinematic variables are the two Dalitz plot variables, namely the invariant masses squared of the $D^{0} p$ and $p \pi^{-}$combinations $M^{2}\left(D^{0} p\right)$ and $M^{2}\left(p \pi^{-}\right)$, and three angles that determine the orientation of the three-body decay plane (figure 2). These angles are defined in the rest frame of the decaying $\Lambda_{b}^{0}$ baryon with the $\hat{x}$ axis given by the direction of the $\Lambda_{b}^{0}$ baryon in the laboratory frame, the polarisation axis $\hat{z}$ given by the cross-product of beam direction and $\hat{x}$ axis, and the $\hat{y}$ axis given by the cross-product of the $\hat{z}$ and $\hat{x}$ axes. The angular variables are the cosine of the polar angle $\cos \vartheta_{p}$, and the azimuthal angle $\varphi_{p}$ of the proton momentum in the reference frame defined above (figure 2(a)), and the angle $\varphi_{D \pi}$ between the $D^{0} \pi^{-}$plane and the plane formed by the proton direction and the polarisation axis $\hat{z}$ (figure $2(\mathrm{~b})$ ).

\subsection{Helicity formalism}

The baseline amplitude fit uses the helicity formalism where the interfering amplitude components are expressed as sequential quasi-two-body decays $\Lambda_{b}^{0} \rightarrow R \pi^{-}, R \rightarrow D^{0} p$ (where $R$ denotes the intermediate resonant or nonresonant state). The decay amplitude for a $\Lambda_{b}^{0}$ baryon with spin projection $\mu$ decaying via an intermediate state $R$ with helicity $\lambda_{R}$ into a final state with proton helicity $\lambda_{p}$ is

$$
\begin{aligned}
& \mathcal{A}_{\mu, \lambda_{R}, \lambda_{p}}\left[M^{2}\left(D^{0} p\right), \theta_{p}, \phi_{p}, \theta_{R}, \phi_{R}\right]= \\
& a_{\lambda_{R}} b_{\lambda_{p}} e^{i\left(\mu-\lambda_{R}\right) \phi_{R}} e^{i\left(\lambda_{R}-\lambda_{p}\right) \phi_{p}} d_{\mu, \lambda_{R}}^{J_{\Lambda_{b}^{0}}}\left(\theta_{R}\right) d_{\lambda_{R} \lambda_{p}}^{J_{R}}\left(\theta_{p}\right) \mathcal{R}\left(M^{2}\left(D^{0} p\right)\right),
\end{aligned}
$$


where $J_{\Lambda_{b}^{0}}=1 / 2$ and $J_{R}$ are the spins of the $\Lambda_{b}^{0}$ baryon and the $R$ state, $d_{\lambda_{1}, \lambda_{2}}^{J}(\theta)$ are the reduced Wigner functions, and $a_{\lambda_{R}}$ and $b_{\lambda_{p}}$ are complex constants (couplings). The mass-dependent complex lineshape $\mathcal{R}\left(M^{2}\right)$ defines the dynamics of the $R$ decay. The angles defining the helicity amplitude are the polar $\left(\theta_{R}\right)$ and azimuthal $\left(\phi_{R}\right)$ angles of the intermediate state $R$ in the reference frame defined above, and the polar $\left(\theta_{p}\right)$ and azimuthal $\left(\phi_{p}\right)$ angles of the final-state proton in the frame where the intermediate state $R$ is at rest and the polar axis points in the direction of $R$ in the $\Lambda_{b}^{0}$ rest frame. All of these angles are functions of the five phase space variables $\Omega$ defined previously and thus do not constitute additional degrees of freedom.

The strong decay $R \rightarrow D^{0} p$ conserves parity, which implies that

$$
b_{\lambda_{p}}=(-1)^{J_{p}+J_{D}-J_{R}} \eta_{R} \eta_{D} \eta_{p} b_{-\lambda_{p}},
$$

where $J_{p}=1 / 2, J_{D}=0$ and $J_{R}$ are the spins of the proton, $D^{0}$ meson and resonance $R$, respectively, and $\eta_{p}=+1, \eta_{D}=-1$ and $\eta_{R}$ are their parities. This relation reduces the number of free parameters in the helicity amplitudes: $\left|b_{\lambda_{p}}\right|$ is absorbed by $a_{\lambda_{R}}$, and each coefficient $a_{\lambda_{R}}$ enters the amplitude multiplied by a factor $\eta_{\lambda_{p}}= \pm 1$. The convention used is

$$
\eta_{\lambda_{p}}= \begin{cases}1 & \text { if } \lambda_{p}=+1 / 2 \\ (-1)^{J_{p}+J_{D}-J_{R}} \eta_{R} \eta_{D} \eta_{p} & \text { if } \lambda_{p}=-1 / 2\end{cases}
$$

As a result, only two couplings $a_{\lambda_{R}}$ remain for each intermediate state $R$, corresponding to its two allowed helicity configurations. The two couplings are denoted for brevity as $a^{ \pm}$.

The amplitude, for fixed $\mu$ and $\lambda_{p}$, after summation over the intermediate resonances $R_{j}$ and their two possible helicities $\lambda_{R_{j}}= \pm 1 / 2$ is

$$
\begin{aligned}
A_{\mu, \lambda_{p}}(\Omega)=e^{i\left(\mu \phi_{R}-\lambda_{p} \phi_{p}\right)} & \sum_{j} \eta_{j, \lambda_{p}}\left[a_{j}^{+} d_{\mu,+1 / 2}^{J_{\Lambda_{b}^{0}}}\left(\theta_{R}\right) d_{+1 / 2, \lambda_{p}}^{J_{R_{i}}}\left(\theta_{p}\right) \mathcal{R}_{j}\left(M^{2}\left(D^{0} p\right)\right)+\right. \\
& \left.a_{j}^{-} d_{\mu,-1 / 2}^{J_{\Lambda_{b}^{0}}}\left(\theta_{R}\right) d_{-1 / 2, \lambda_{p}}^{J_{R_{i}}}\left(\theta_{p}\right) \mathcal{R}_{j}\left(M^{2}\left(D^{0} p\right)\right) e^{i\left(\phi_{R}-\phi_{p}\right)}\right] .
\end{aligned}
$$

To obtain the decay probability density, the amplitudes corresponding to different polarisations of the initial- and final-state particles have to be summed up incoherently. The $\Lambda_{b}^{0}$ baryons produced in $p p$ collisions can only have polarisation transverse to the production plane, i.e. along the $\hat{z}$ axis. The longitudinal component is forbidden due to parity conservation in the strong processes that dominate $\Lambda_{b}^{0}$ production. In this case, the probability density function (PDF) of the kinematic variables that characterise the decay of a $\Lambda_{b}^{0}$ with the transverse polarisation $P_{z}$, after summation over $\mu$ and $\lambda_{p}$, is proportional to

$$
p\left(\Omega, P_{z}\right)=\sum_{\mu, \lambda_{p}= \pm 1 / 2}\left(1+2 \mu P_{z}\right)\left|A_{\mu, \lambda_{p}}(\Omega)\right|^{2} .
$$

Equations (3.4) and (3.5) can be combined to yield the simplified expression:

$$
p\left(\Omega, P_{z}\right)=\sum_{n=0}^{2 J_{\max }} p_{n}\left(M^{2}\left(D^{0} p\right)\right) \cos \left(n \theta_{p}\right)+P_{z} \cos \theta_{R} \sum_{n=0}^{2 J_{\max }} q_{n}\left(M^{2}\left(D^{0} p\right)\right) \cos \left(n \theta_{p}\right),
$$


where $J_{\max }$ is the highest spin among the intermediate resonances and $p_{n}$ and $q_{n}$ are functions of only $M^{2}\left(D^{0} p\right)$. As a consequence, $p\left(\Omega, P_{z}\right)$ does not depend on the azimuthal angles $\phi_{p}$ and $\phi_{R}$. Dependence on the angle $\theta_{R}$ appears only if the $\Lambda_{b}^{0}$ is polarised. In the unpolarised case the density depends only on the internal degrees of freedom $M^{2}\left(D^{0} p\right)$ and $\theta_{p}$ (which in turn can be expressed as a function of the other Dalitz plot variable, $\left.M^{2}\left(p \pi^{-}\right)\right)$. Moreover, after integration over the angle $\theta_{R}$, the dependence on polarisation cancels if the detection efficiency is symmetric over $\cos \theta_{R}$. Since $\Lambda_{b}^{0}$ polarisation in $p p$ collisions is measured to be small $\left(P_{z}=0.06 \pm 0.07 \pm 0.02\right.$, [46] $)$ and the efficiency is highly symmetric in $\cos \theta_{R}$, the effects of polarisation can safely be neglected in the amplitude analysis, and only the Dalitz plot variables $\omega=\left(M^{2}\left(D^{0} p\right), M^{2}\left(p \pi^{-}\right)\right)$need to be used to describe the probability density $p(\omega)$ of the decay. The density $p(\omega)$ is given by eq. (3.5) with $P_{z}=0$ such that no dependence on the angles $\vartheta_{p}, \varphi_{p}$ or $\varphi_{D \pi}$ remains.

Up to this point, the formalism has assumed that resonances are present only in the $D^{0} p$ channel. While in the case of $\Lambda_{b}^{0} \rightarrow D^{0} p \pi^{-}$decays the regions of phase space with contributions from $D^{0} p$ and $p \pi^{-}$resonances are generally well separated, there is a small region where they can overlap, and thus interference between resonances in the two channels has to be taken into account. In the helicity formalism, the proton spin-quantisation axes are different for the helicity amplitudes corresponding to $D^{0} p$ and $p \pi^{-}$resonances [8]: they are parallel to the proton direction in the $D^{0} p$ and $p \pi^{-}$rest frames, and are thus antiparallel to the $\pi^{-}$and $D^{0}$ momenta, respectively. The rotation angle between the two spin-quantisation axes is given by

$$
\cos \theta_{\mathrm{rot}}=\frac{\left(\vec{p}_{\pi^{-}}^{(p)} \cdot \vec{p}_{D^{0}}^{(p)}\right)}{\left.\left|\vec{p}_{\pi^{-}}^{(p)}\right| \mid \vec{p}_{D^{0}}^{(p)}\right) \mid},
$$

where $\vec{p}_{\pi^{-}}^{(p)}$ and $\vec{p}_{D^{0}}^{(p)}$ are the momenta of the $\pi^{-}$and $D^{0}$ mesons, respectively, in the proton rest frame.

If the proton spin-quantisation axis is chosen with respect to the $D^{0} p$ resonances and the helicity basis is denoted as $\left|\lambda_{p}\right\rangle$, the helicity states $\left|\lambda_{p}^{\prime}\right\rangle$ corresponding to $p \pi^{-}$states are

$$
\left|\lambda_{p}^{\prime}\right\rangle=\sum_{\lambda_{p}^{\prime}= \pm 1 / 2} d_{\lambda_{p}, \lambda_{p}^{\prime}}^{1 / 2}\left(\theta_{\mathrm{rot}}\right)\left|\lambda_{p}\right\rangle
$$

and thus the additional terms in the amplitude (eq. (3.4)) related to the $p \pi^{-}$channel are expressed as

$$
\begin{aligned}
A_{\mu, \lambda_{p}}^{\left(p \pi^{-}\right)}(\Omega)= & \sum_{\lambda_{p}^{\prime}= \pm 1 / 2} d_{\lambda_{p}, \lambda_{p}^{\prime}}^{1 / 2}\left(\theta_{\mathrm{rot}}\right) e^{i\left(\mu \phi_{R}^{\prime}-\lambda_{p}^{\prime} \phi_{p}^{\prime}\right)} \sum_{j} \eta_{j, \lambda_{p}^{\prime}} \times \\
& {\left[a_{j}^{+} d_{\mu,+1 / 2}^{J_{\Lambda_{b}^{0}}}\left(\theta_{R}^{\prime}\right) d_{+1 / 2, \lambda_{p}^{\prime}}^{J_{R_{i}}}\left(\theta_{p}^{\prime}\right) \mathcal{R}_{j}\left(M^{2}\left(p \pi^{-}\right)\right)+\right.} \\
& \left.a_{j}^{-} d_{\mu,-1 / 2}^{J_{\Lambda_{b}^{0}}}\left(\theta_{R}^{\prime}\right) d_{-1 / 2, \lambda_{p}^{\prime}}^{J_{R_{i}}}\left(\theta_{p}^{\prime}\right) \mathcal{R}_{j}\left(M^{2}\left(p \pi^{-}\right)\right) e^{i\left(\phi_{R}^{\prime}-\phi_{p}^{\prime}\right)}\right],
\end{aligned}
$$

where the angles $\theta_{p}^{\prime}, \phi_{p}^{\prime}, \theta_{R}^{\prime}$ and $\phi_{R}^{\prime}$ are defined in a similar way as $\theta_{p}, \phi_{p}, \theta_{R}$ and $\phi_{R}$, but with the intermediate state $R$ in the $p \pi^{-}$channel. 


\subsection{Resonant and nonresonant lineshapes}

The part of the amplitude that describes the dynamics of the quasi-two-body decay, $\mathcal{R}\left(M^{2}\right)$, is given by one of the following functions. Resonances are parametrised with relativistic Breit-Wigner lineshapes multiplied by angular barrier terms and corrected by BlattWeisskopf form factors [47]:

$$
\mathcal{R}_{\mathrm{BW}}\left(M^{2}\right)=\left[\frac{q(M)}{q_{0}}\right]^{L_{\Lambda_{b}^{0}}}\left[\frac{p(M)}{p_{0}}\right]^{L_{R}} \frac{F_{\Lambda_{b}^{0}}\left(M, L_{\Lambda_{b}^{0}}\right) F_{R}\left(M, L_{R}\right)}{m_{R}^{2}-M^{2}-i m_{R} \Gamma(M)},
$$

with mass-dependent width $\Gamma(M)$ given by

$$
\Gamma(M)=\Gamma_{0}\left[\frac{p(M)}{p_{0}}\right]^{2 L_{R}+1} \frac{m_{R}}{M} F_{R}^{2}\left(M, L_{R}\right),
$$

where $m_{R}$ and $\Gamma_{0}$ are the pole parameters of the resonance. The Blatt-Weisskopf form factors for the resonance, $F_{R}\left(M, L_{R}\right)$, and for the $\Lambda_{b}^{0}, F_{\Lambda_{b}^{0}}\left(M, L_{\Lambda_{b}^{0}}\right)$, are parametrised as

$$
F_{R, \Lambda_{b}^{0}}(M, L)=\left\{\begin{array}{ll}
1 & L=0 \\
\sqrt{\frac{1+z_{0}^{2}}{1+z^{2}(M)}} & L=1 \\
\sqrt{\frac{9+3 z_{0}^{2}+z_{0}^{4}}{9+3 z^{2}(M)+z^{4}(M)}} & L=2 \\
\sqrt{\frac{225+45 z_{0}^{2}+6 z_{0}^{4}+z_{0}^{6}}{225+45 z^{2}(M)+6 z^{4}(M)+z^{6}(M)}} & L=3
\end{array},\right.
$$

where the definitions of the terms $z(M)$ and $z_{0}$ depend on whether the form factor for the resonance $R$ or for the $\Lambda_{b}^{0}$ is being considered. For $R$ these terms are given by $z(M)=$ $p(M) d$ and $z_{0}=p_{0} d$, where $p(M)$ is the centre-of-mass momentum of the decay products in the two-body decay $R \rightarrow D^{0} p$ with the mass of the resonance $R$ equal to $M, p_{0} \equiv p\left(m_{R}\right)$, and $d$ is a radial parameter taken to be $1.5 \mathrm{GeV}^{-1}$. For $\Lambda_{b}^{0}$ the respective functions are $z(M)=q(M) d$ and $z_{0}=q_{0} d$, where $q(M)$ is the centre-of-mass momentum of decay products in the two-body decay $\Lambda_{b}^{0} \rightarrow R \pi^{-}, q_{0}=q\left(m_{R}\right)$, and $d=5.0 \mathrm{GeV}^{-1}$. The analysis is very weakly sensitive to the values of $d$, and these are varied in a wide range for assessing the associated systematic uncertainty (section 9.2).

The mass-dependent width and form factors depend on the orbital angular momenta of the two-body decays. For the weak decay of the $\Lambda_{b}^{0}$, the minimum possible angular momentum $L_{\Lambda_{b}^{0}}=J-1 / 2$ (where $J$ is the spin of the resonance) is taken, while for the strong decay of the intermediate resonance, the angular momentum $L_{R}$ is fully determined by the parity of the resonance, $P=(-1)^{L_{R}+1}$, and conservation of angular momentum, which requires $L_{R}=J \pm 1 / 2$.

Two parametrisations are used for nonresonant amplitudes: exponential and polynomial functions. The exponential nonresonant lineshape [48] used is

$$
\mathcal{R}_{\mathrm{NRexp}}\left(M^{2}\right)=\left[\frac{q(M)}{q_{0}}\right]^{L_{\Lambda_{b}^{0}}}\left[\frac{p(M)}{p_{0}}\right]^{L_{R}} e^{-\alpha M^{2}},
$$

where $\alpha$ is a shape parameter. The polynomial nonresonant lineshape [49] used is

$$
\mathcal{R}_{\text {NRpoly }}\left(M^{2}\right)=\left[\frac{q(M)}{q_{0}}\right]^{L_{\Lambda_{b}^{0}}}\left[\frac{p(M)}{p_{0}}\right]^{L_{R}}\left(a_{2} \Delta M^{2}+a_{1} \Delta M+a_{0}\right)
$$


where $\Delta M=M-M_{0}$, and $M_{0}$ is a constant that is chosen to minimise the correlations between the coefficients $a_{i}$ when they are treated as free parameters. In the case of the $D^{0} p$ amplitude fit, $M_{0}$ is chosen to be near the middle of the fit range, $M_{0} \equiv 2.88 \mathrm{GeV}$. In both the exponential and the polynomial parametrisations, $M_{0}$ also serves as the resonance mass parameter in the definition of $p_{0}$ and $q_{0}$ in the angular barrier terms. Note that in ref. [49] the polynomial form was introduced to describe the slow variations of a nonresonant amplitude across the large phase space of charmless $B$ decays, and thus the parameters $a_{i}$ were defined as complex constants to allow slow phase motion over the wide range of invariant masses. In the present analysis, the phase space is much more constrained and no significant phase rotation is expected for the nonresonant amplitudes. The coefficients $a_{i}$ thus are taken to be real.

To study the resonant nature of the $D^{0} p$ states, model-independent parametrisations of the lineshape are used. One approach used here consists of interpolation with cubic splines, done independently for the real and imaginary parts of the amplitude (referred to as the "complex spline" lineshape) [50]. The free parameters of such a fit are the real $\operatorname{Re}\left(\mathcal{R}_{i}\right)$ and imaginary $\operatorname{Im}\left(\mathcal{R}_{i}\right)$ parts of the amplitude at the spline knot positions. Alternatively, to assess the significance of the complex phase rotation in a model-independent way, a splineinterpolated shape is used in which the imaginary parts of the amplitude at all knots are fixed to zero ("real spline").

\subsection{Fitting procedure}

An unbinned maximum likelihood fit is performed in the two-dimensional phase space $\omega=\left(M^{2}\left(D^{0} p\right), M^{2}\left(p \pi^{-}\right)\right)$. Defining $\mathcal{L}$ as the likelihood function, the fit minimises

$$
-2 \ln \mathcal{L}=-2 \sum_{i=1}^{N} \ln p_{\text {tot }}\left(\omega_{i}\right),
$$

where the summation is performed over all candidates in the data sample and $p_{\text {tot }}$ is the normalised PDF. It is given by

$$
p_{\text {tot }}(\omega)=p(\omega) \epsilon(\omega) \frac{n_{\text {sig }}}{\mathcal{N}}+p_{\text {bck }}(\omega) \frac{n_{\text {bck }}}{\mathcal{N}_{\text {bck }}},
$$

where $p(\omega)$ is the signal PDF, $p_{\text {bck }}(\omega)$ is the background PDF, $\epsilon(\omega)$ is the efficiency, and $\mathcal{N}$ and $\mathcal{N}_{\text {bck }}$ are the signal and background normalisations:

$$
\mathcal{N}=\int_{\mathcal{D}} p(\omega) \epsilon(\omega) d \omega,
$$

and

$$
\mathcal{N}_{\text {bck }}=\int_{\mathcal{D}} p_{\text {bck }}(\omega) d \omega,
$$

where the integrals are taken over the part of the phase space $\mathcal{D}$ used in the fit (section 5), and $n_{\text {sig }}$ and $n_{\text {bck }}$ are the numbers of signal and background events in the signal region, respectively, evaluated from a fit to the $M\left(D^{0} p \pi^{-}\right)$invariant mass distribution. The normalisation integrals are calculated numerically using a fine grid with $400 \times 400$ cells in the 
baseline fits; the numerical uncertainty is negligible compared with the other uncertainties in the analysis.

\subsection{Fit parameters and fit fractions}

The free parameters in the fit are the couplings $a^{ \pm}$for each of the amplitude components and certain parameters of the lineshapes (such as the masses and/or widths of the resonant states, or shape parameters of the nonresonant lineshapes). Since the overall normalisation of the density is arbitrary, one of the couplings can be set to unity. In this analysis, the convention $a^{+} \equiv 1$ for the $\Lambda_{c}(2880)^{+}$state is used. Additionally, the amplitudes corresponding to different helicity states of the initial- and final-state particles are added incoherently, so that the relative phase between $a^{+}$and $a^{-}$for one of the contributions is arbitrary. The convention $\operatorname{Im}\left(a^{-}\right) \equiv 0$ for the $\Lambda_{c}(2880)^{+}$is used.

The definitions of the polynomial and spline-interpolated shapes already contain terms that characterise the relative magnitudes of the corresponding amplitudes. The couplings for them are defined in such a way as to remove the additional degree of freedom from the fit. For the polynomial and real spline lineshapes, the following couplings are used:

$$
a^{+}=r e^{i \phi_{+}}, \quad a^{-}=(1-r) e^{i \phi_{-}},
$$

where $r, \phi_{+}$and $\phi_{-}$are free parameters. For the complex spline lineshape, a similar parametrisation is used with $\phi_{+}$fixed to zero, since the complex phase is already included in the spline definition.

The observable decay density for an unpolarised particle in the initial state does not allow each polarisation amplitude to be obtained independently. As a result, the couplings $a^{ \pm}$in the fit can be strongly correlated. However, the size of each contribution can be characterised by its spin-averaged fit fraction

$$
\mathcal{F}_{i}=\frac{\sum_{\mu, \lambda_{p}= \pm 1 / 2} \int_{\mathcal{D}}\left|A_{\mu, \lambda_{p}}^{(i)}(\omega)\right|^{2} d \omega}{\sum_{\mu, \lambda_{p}= \pm 1 / 2} \int_{\mathcal{D}}\left|\sum_{i} A_{\mu, \lambda_{p}}^{(i)}(\omega)\right|^{2} d \omega} .
$$

If all the components correspond to partial waves with different spin-parities, the sum of the spin-averaged fit fractions will be 100\%; otherwise it can differ from $100 \%$ due to interference effects. The statistical uncertainties on the fit fractions are obtained from ensembles of pseudoexperiments.

\subsection{Evaluation of fit quality}

To assess the goodness of each fit, a $\chi^{2}$ value is calculated by summing over the bins of the two-dimensional Dalitz plot. Since the amplitude is highly non-uniform and a meaningful $\chi^{2}$ test requires a certain minimum number of entries in each bin, an adaptive binning method is used to ensure that each bin contains at least 20 entries in the data.

Since the fit itself is unbinned, some information is lost by the binning. The number of degrees of freedom for the $\chi^{2}$ test in such a case is not well defined. The effective number 
of degrees of freedom ( ndf $_{\text {eff }}$ ) should be in the range $N_{\text {bins }}-N_{\text {par }}-1 \leq \operatorname{ndf}_{\text {eff }} \leq N_{\text {bins }}-1$, where $N_{\text {bins }}$ is the number of bins and $N_{\text {par }}$ is the number of free parameters in the fit. For each fit, ndf $_{\text {eff }}$ is obtained from ensembles of pseudoexperiments by requiring that the probability value for the $\chi^{2}$ distribution with $\mathrm{ndf}_{\text {eff }}$ degrees of freedom, $P\left(\chi^{2}, \mathrm{ndf}_{\text {eff }}\right)$, is distributed uniformly.

Note that when two fits with different models have similar binned $\chi^{2}$ values, it does not necessarily follow that both models describe the data equally well. Since the bins in regions with low population density have large area, the binning can obscure features that could discriminate between the models. This information is preserved in the unbinned likelihood. Thus, discrimination between fit models is based on the difference $\Delta \ln \mathcal{L}$, the statistical significance of which is determined using ensembles of pseudoexperiments. The binned $\chi^{2}$ serves as a measure of the fit quality for individual models and is not used to discriminate between them.

\section{Signal selection}

The analysis uses the decay $\Lambda_{b}^{0} \rightarrow D^{0} p \pi^{-}$, where $D^{0}$ mesons are reconstructed in the final state $K^{-} \pi^{+}$. The selection of $\Lambda_{b}^{0}$ candidates is performed in three stages: a preliminary selection, a kinematic fit, and a final selection. The preliminary selection uses loose criteria on the kinematic and topological properties of the $\Lambda_{b}^{0}$ candidate. All tracks forming a candidate, as well as the $\Lambda_{b}^{0}$ and $D^{0}$ vertices, are required to be of good quality and be separated from every PV in the event. The separation from a PV is characterised by a quantity $\chi_{\mathrm{IP}}^{2}$, defined as the increase in the vertex-fit $\chi^{2}$ when the track (or combination of tracks corresponding to a short-lived particle) is included into the vertex fit. The tracks forming a $D^{0}$ candidate are required to be positively identified as a pion and a kaon, and the $\Lambda_{b}^{0}$ and $D^{0}$ decay vertices are required to be downstream of their production vertices. All of the tracks are required to have no associated hits in the muon detector.

For candidates passing this initial selection, a kinematic fit is performed [51]. Constraints are imposed that the $\Lambda_{b}^{0}$ and $D^{0}$ decay products originate from the corresponding vertices, that the $\Lambda_{b}^{0}$ candidate originate from its associated PV (the one with the smallest value of $\chi_{\mathrm{IP}}^{2}$ for the $\Lambda_{b}^{0}$ ), and that the mass of the $D^{0}$ candidate be equal to its known value [23]. The kinematic fit is required to converge with a good $\chi^{2}$, and the mass of the $\Lambda_{b}^{0}$ candidate after the fit is required to be in the range $5400-5900 \mathrm{MeV}$. To suppress background from charmless $\Lambda_{b}^{0} \rightarrow p K^{-} \pi^{+} \pi^{-}$decays, the decay time significance of the $D^{0}$ candidate obtained after the fit is required to be greater than one standard deviation. To improve the resolution of the squared invariant masses $M^{2}\left(D^{0} p\right)$ and $M^{2}\left(p \pi^{-}\right)$entering the amplitude fit, the additional constraint that the invariant mass of the $D^{0} p \pi^{-}$combination be equal to the known $\Lambda_{b}^{0}$ mass [23] is applied when calculating these variables.

After the initial selection, the background in the region of the $\Lambda_{b}^{0} \rightarrow D^{0} p \pi^{-}$signal is dominated by random combinations of tracks. The final selection is based on a boosted decision tree (BDT) algorithm $[52,53]$ designed to separate signal from this background. The selection is trained using simulated $\Lambda_{b}^{0} \rightarrow D^{0} p \pi^{-}$events generated uniformly across the phase space as the signal sample, and the sample of opposite-flavour $\bar{D}^{0} p \pi^{-}, \bar{D}^{0} \rightarrow K^{+} \pi^{-}$ 
combinations from data as background. In total, 12 discriminating variables are used in the BDT selection: the $\chi^{2}$ of the kinematic fit, the angle between the momentum and the direction of flight of the $\Lambda_{b}^{0}$ candidate, the $\chi^{2}$ of the $\Lambda_{b}^{0}$ and $D^{0}$ vertex fits, the lifetime significance of the $D^{0}$ candidate with respect to the $\Lambda_{b}^{0}$ vertex, the $\chi_{\mathrm{IP}}^{2}$ of the final-state tracks and the $D^{0}$ candidate, and the particle identification (PID) information of the proton and pion tracks from the $\Lambda_{b}^{0}$ vertex. Due to differences between simulation and data, corrections are applied to all the variables from the simulated sample used in the BDT training, except for the PID variables. These corrections are typically about $10 \%$ and are obtained from a large and clean sample of $\Lambda_{b}^{0} \rightarrow \Lambda_{c}^{+} \pi^{-}$decays. The simulated proton and pion PID variables are replaced with values generated using distributions obtained from calibration samples of $D^{*+} \rightarrow D^{0} \pi^{+}$and $\Lambda_{c}^{+} \rightarrow p K^{-} \pi^{+}$decays in data. For these calibration samples, the four-dimensional distributions of PID variable, $p_{\mathrm{T}}, \eta$ and the track multiplicity of the event are described using a nonparametric kernel-based procedure [54]. The resulting distributions are used to generate PID variables for each pion or proton track given its $p_{\mathrm{T}}, \eta$ and the track multiplicity in the simulated event.

The BDT requirement is chosen such that the fraction of background in the signal region used for the subsequent amplitude fit, $\left|M\left(D^{0} p \pi^{-}\right)-m\left(\Lambda_{b}^{0}\right)\right|<30 \mathrm{MeV}$, does not exceed 15\%. This corresponds to a signal efficiency of $66 \%$ and a background rejection of $96 \%$ with respect to the preliminary selection. After all selection requirements are applied, fewer than $1 \%$ of selected events contain a second candidate. All multiple candidates are retained; the associated systematic uncertainty is negligible.

\section{$5 \quad$ Fit regions and event yields}

The Dalitz plot of selected events, without background subtraction or efficiency correction, in the signal $D^{0} p \pi^{-}$invariant mass range defined in section 4 is shown in figure 3(a). The part of the phase space near the $D^{0} p$ threshold that contains contributions from $\Lambda_{c}^{*+}$ resonances is shown in figure 3(b). The latter uses $M\left(D^{0} p\right)$ as the horizontal axis instead of $M^{2}\left(D^{0} p\right)$.

In figure 3 , the four amplitude fit regions of the $\Lambda_{b}^{0} \rightarrow D^{0} p \pi^{-}$phase space are indicated. These are denoted regions $1-4$. Region $1, M\left(D^{0} p\right)>3 \mathrm{GeV}$ and $M\left(p \pi^{-}\right)>2 \mathrm{GeV}$, is the part of the phase space that does not include resonant contributions and is used only to constrain the nonresonant $p \pi^{-}$amplitude in the $D^{0} p$ regions. Region 2, $2.86<M\left(D^{0} p\right)<2.90 \mathrm{GeV}$, contains the well-known $\Lambda_{c}(2880)^{+}$state and is used to measure its parameters and to constrain the slowly varying amplitude underneath it in a model-independent way. The fit in region 3 near the $D^{0} p$ threshold, $M\left(D^{0} p\right)<2.90 \mathrm{GeV}$, provides additional information about the slowly-varying $D^{0} p$ amplitude. Finally, the fit in region $4, M\left(D^{0} p\right)<3.00 \mathrm{GeV}$, which includes the $\Lambda_{c}(2940)^{+}$state, gives information about the properties of this resonance and the relative magnitudes of the resonant and nonresonant contributions. Note that region 2 is fully contained in region 3 , while region 3 is fully contained in region 4 .

The signal and background yields in each region are obtained from extended unbinned maximum likelihood fits of the $D^{0} p \pi^{-}$invariant mass distribution in the range 

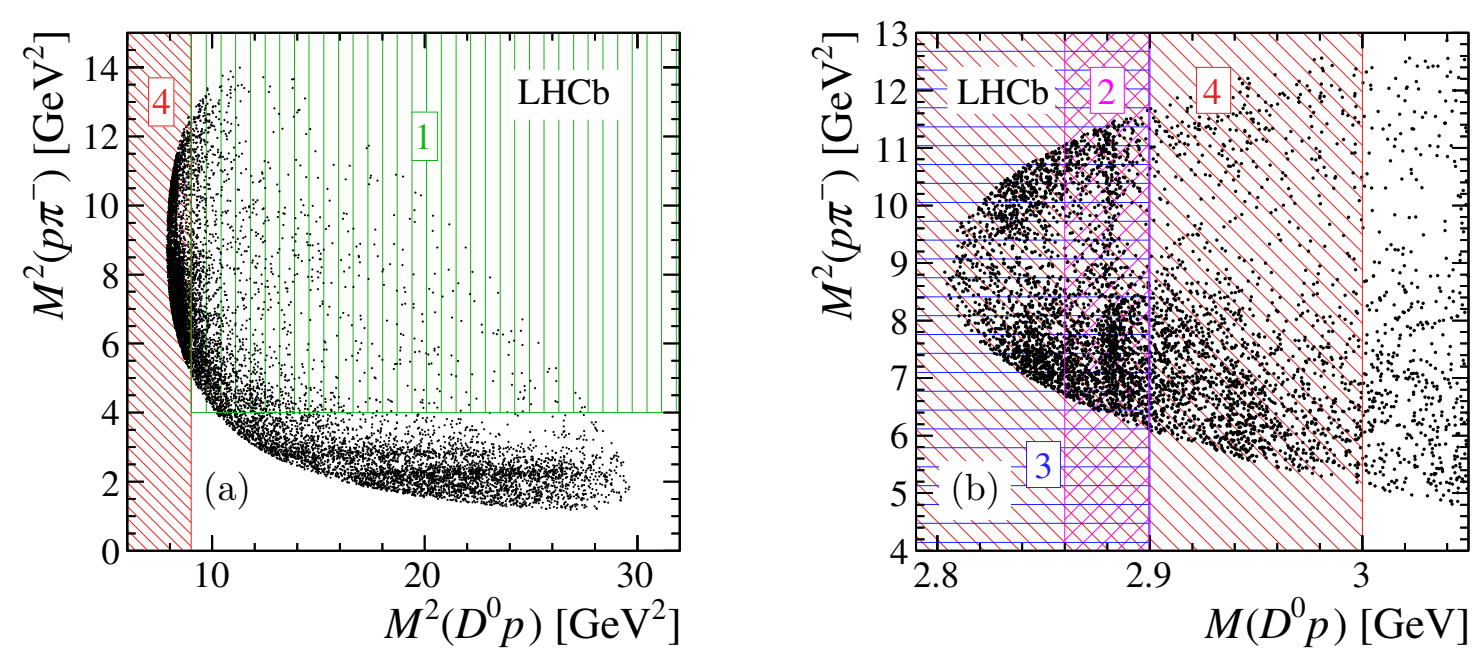

Figure 3. Distributions of $\Lambda_{b}^{0} \rightarrow D^{0} p \pi^{-}$candidates in data: (a) the full Dalitz plot as a function of $M^{2}\left(D^{0} p\right)$ and $M^{2}\left(p \pi^{-}\right)$, and (b) the part of the phase space including the resonances in the $D^{0} p$ channel (note the change in variable on the horizontal axis). The distributions are neither background-subtracted nor efficiency-corrected. The hatched areas 1-4 are described in the text.

5400-5900 MeV. The fit model includes the signal component, a contribution from random combinations of tracks (combinatorial background) and the background from partially reconstructed $\Lambda_{b}^{0} \rightarrow D^{* 0} p \pi^{-}$decays (where $D^{* 0}$ decays into $D^{0} \pi^{0}$ or $D^{0} \gamma$ and the $\pi^{0}$ or $\gamma$ are not included in the reconstruction).

The signal component is modelled as the sum of two Crystal Ball functions [55] with the same most probable value and power-law tails on both sides. All parameters of the model are fixed from simulation except for the peak position and a common scale factor for the core widths, which are floated in the fit to data. The combinatorial background is parametrised by an exponential function, and the partially reconstructed background is described by a bifurcated Gaussian distribution. The shape parameters of the background distributions are free parameters of the fit.

The results of the fit for candidates in the entire $D^{0} p \pi^{-}$phase space are shown in figure 4 . The background and signal yields in the entire $D^{0} p \pi^{-}$phase space, as well as in the regions used in the amplitude fit, are given in table 1.

\section{Efficiency variation over the Dalitz plot}

The same sample of simulated events as in the selection training (section 4) is used to determine the variation of the efficiency across the Dalitz plot. The sample is generated uniformly in the decay phase space and consists of approximately $8 \times 10^{4} \Lambda_{b}^{0} \rightarrow D^{0} p \pi^{-}$ events satisfying the selection requirements. Each simulated event is assigned a weight, derived from control samples of data, to correct for known differences in track reconstruction and hardware trigger efficiency between data and simulation. Since the PID variables in the sample are replaced by those generated from calibration data, the efficiency of PID requirements is included in the efficiency calculation and does not need to be treated separately. 


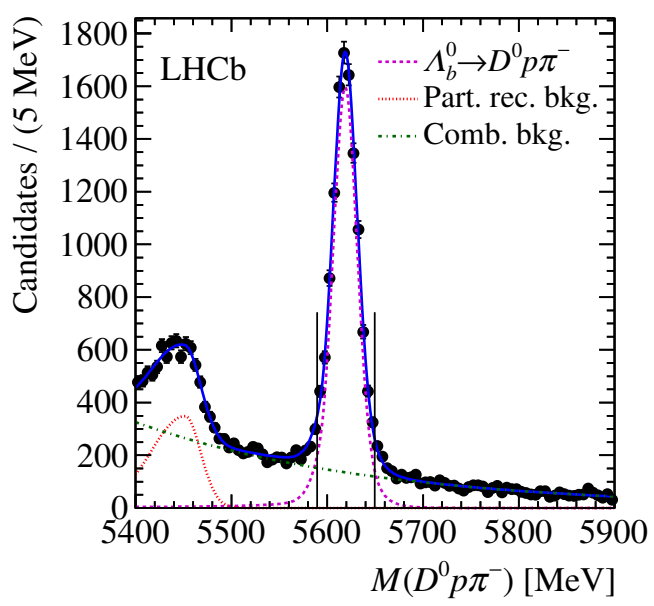

Figure 4. Invariant mass distribution for the $D^{0} p \pi^{-}$candidates in the entire $D^{0} p \pi^{-}$phase space. The blue solid line is the fit result. Signal, partially reconstructed and combinatorial background components are shown with different line styles. Vertical lines indicate the boundaries of the signal region used in the amplitude fit.

\begin{tabular}{|l|ccccc|}
\hline \multirow{2}{*}{ Yield } & \multicolumn{5}{|c|}{ Phase space region } \\
\cline { 2 - 6 } & Full & 1 & 2 & 3 & 4 \\
\hline$\Lambda_{b}^{0} \rightarrow D^{0} p \pi^{-}$ & $11212 \pm 126$ & $2250 \pm 61$ & $1674 \pm 46$ & $3141 \pm 63$ & $4750 \pm 79$ \\
Combinatorial & $14024 \pm 224$ & $4924 \pm 132$ & $968 \pm 78$ & $2095 \pm 96$ & $4188 \pm 127$ \\
Partially rec. & $4106 \pm 167$ & $1344 \pm 96$ & $321 \pm 64$ & $691 \pm 75$ & $1204 \pm 96$ \\
\hline Signal in box & 10233 & 2061 & 1500 & 2803 & 4261 \\
Background in box & 1616 & 598 & 89 & 192 & 427 \\
\hline
\end{tabular}

Table 1. Results of the fits to the $\Lambda_{b}^{0} \rightarrow D^{0} p \pi^{-}$mass distribution in the entire $\Lambda_{b}^{0} \rightarrow D^{0} p \pi^{-}$phase space and in the four phase space regions used in the amplitude fits. The signal and background yields for the full $M\left(D^{0} p \pi^{-}\right)$range, as well as for the amplitude fit region $\left|M\left(D^{0} p \pi^{-}\right)-m\left(\Lambda_{b}^{0}\right)\right|<$ $30 \mathrm{MeV}$ ("box"), are reported.

The Dalitz plot efficiency profile is calculated separately for two disjoint sets of candidates, defined according to whether the hardware trigger was activated by one of the $\Lambda_{b}^{0}$ decay products or by other particles in the event. For each of those samples, a kernelbased density estimation procedure with a correction for boundary effects [54] is used to obtain a description of the relative efficiency as a function of the Dalitz plot variables. The overall efficiency is then given by the average of the two profiles, weighted according to the ratio of yields of the two classes of events in data. The resulting profile is shown in figure 5(a). The normalisation of the efficiency profile used in the amplitude fit likelihood (eqs. (3.15) and (3.16)) does not affect the result. The efficiency profile shown in figure 5(a) is normalised such that the average efficiency over the phase space is equal to unity. 

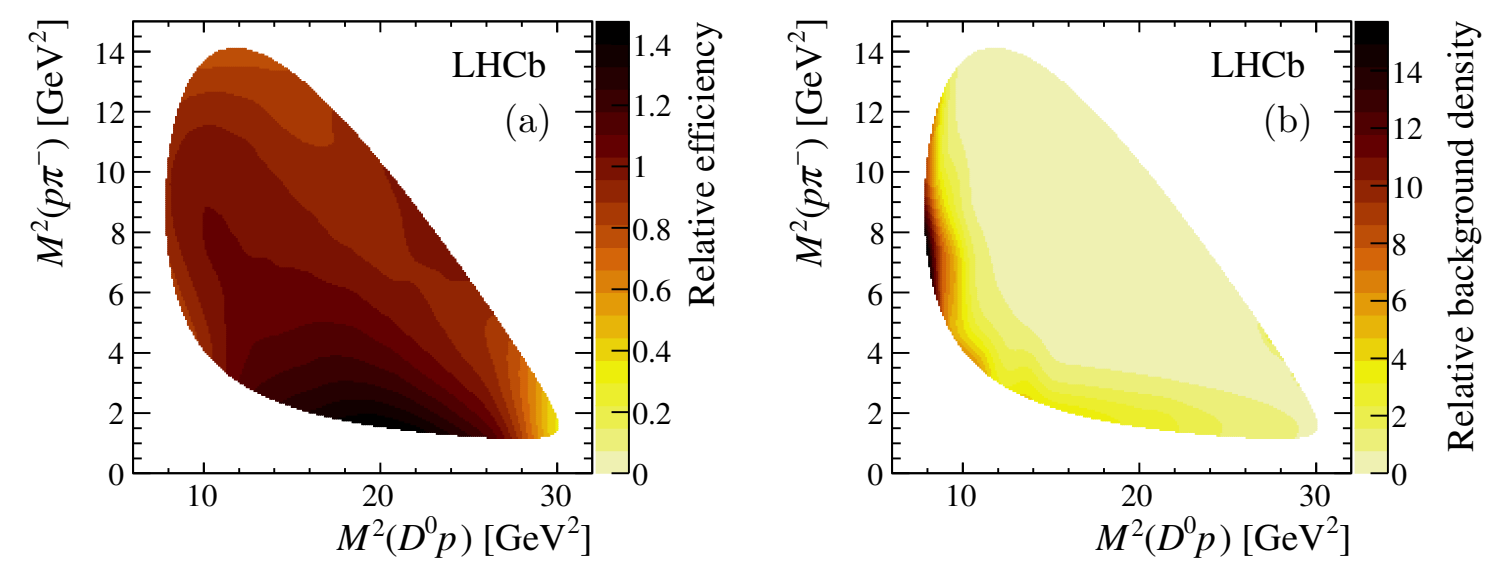

Figure 5. (a) Relative selection efficiency and (b) background density over the $\Lambda_{b}^{0} \rightarrow D^{0} p \pi^{-}$phase space. The normalisations are such that the average over the phase space is unity.

\section{Background distribution}

Background in the vicinity of the $\Lambda_{b}^{0} \rightarrow D^{0} p \pi^{-}$invariant mass peak is dominated by random combinations of $D^{0}$ mesons, proton, and pion tracks. To determine the background shape as a function of Dalitz plot variables $M^{2}\left(D^{0} p\right)$ and $M^{2}\left(p \pi^{-}\right)$, the $\Lambda_{b}^{0}$ mass sidebands are used: $5500<M\left(D^{0} p \pi^{-}\right)<5560 \mathrm{MeV}$ and $5680<M\left(D^{0} p \pi^{-}\right)<5900 \mathrm{MeV}$. The same procedure is applied to the opposite-flavour $\bar{D}^{0} p \pi^{-}$sample to verify that the background shape in the mass sidebands is representative of that in the signal window. Good agreement is found.

The background distribution as a function of the Dalitz plot variables is estimated using a Gaussian mixture model, describing the background as a sum of several twodimensional Gaussian distributions, whose parameters are allowed to vary in the fit. For the limited-size sample of background events this approach appears more suitable than a kernel-based technique. The parametrisation is obtained using an iterative procedure where Gaussian components are added to the model one by one; at each iteration the parameters of all components are adjusted using an unbinned maximum likelihood fit. The result of the procedure is shown in figure 5(b). The baseline parametrisation is a sum of 25 two-dimensional Gaussian components. The normalisation of the background density used in the fit is arbitrary; for the purposes of illustration in figure 5(b) it is set such that the average density across the phase space is unity.

\section{Effect of momentum resolution}

Finite momentum resolution smears the structures in the Dalitz plot. The use of the kinematic fit with $\Lambda_{b}^{0}$ and $D^{0}$ mass constraints significantly improves the resolution near the edges of the phase space, but less so in the central region. The only structure in the $\Lambda_{b}^{0} \rightarrow D^{0} p \pi^{-}$amplitude that is expected to be affected by the finite resolution is the resonance $\Lambda_{c}(2880)^{+}$, which has a natural width of approximately $6 \mathrm{MeV}$. Therefore, only 
the $M\left(D^{0} p\right)$ resolution is considered, and is obtained from a sample of simulated events by comparing the generated and reconstructed values of $M\left(D^{0} p\right)$. The width of the resolution function at $M\left(D^{0} p\right)=2.88 \mathrm{GeV}$ is $1.1 \mathrm{MeV}$, i.e. significantly smaller than the natural width of the $\Lambda_{c}(2880)^{+}$. However, simulation shows that neglecting the resolution would lead to a bias on the $\Lambda_{c}(2880)^{+}$width of about $10 \%$. Therefore, the $M\left(D^{0} p\right)$ resolution is taken into account in the fit by convolving the signal PDF with a Gaussian resolution function, where the width of the Gaussian is a function of $M\left(D^{0} p\right)$.

\section{Amplitude analysis}

The amplitude fit is performed in the four phase space regions defined in figure 3. This approach has been chosen instead of performing the fit to the entire Dalitz plot since the amplitude contains many unexplored contributions. The full fit would include too many degrees of freedom and a very large range of systematic variations would need to be considered. Instead, the fit is first performed around the well-known resonance $\Lambda_{c}(2880)^{+}$and then the fitting region is gradually extended to include a larger portion of the phase space.

\subsection{Fit in the nonresonant region}

The fit in region 1, where no significant resonant contributions are expected, provides constraints on the high-mass behaviour of the $p \pi^{-}$amplitude, and thus on the $p \pi^{-}$partial waves in the $D^{0} p$ fit regions. The fit model includes four exponential nonresonant components (eq. (3.13)) in each of the $D^{0} p$ and $p \pi^{-}$spectra, corresponding to the four combinations of spin $(1 / 2$ and $3 / 2)$ and parity (negative and positive). Since there is no reference amplitude with known parity in this region, there is an ambiguity: all parities can be reversed simultaneously without changing the amplitude. The shape parameters $\alpha$ of all eight nonresonant components are varied in the fit.

The projections of the fitted data are shown in figure 6 . The fitted $p \pi^{-}$amplitude is extrapolated into the regions $2-4$ of the $\Lambda_{b}^{0} \rightarrow D^{0} p \pi^{-}$phase space using the fitted helicity distributions. The estimated contributions of the $p \pi^{-}$nonresonant components in the $D^{0} p$ mass regions are given in table 2 and compared with the total numbers of signal events in those regions. They amount to less than $1 \%$ of the signal yield for the regions 2 and 3, and around $1.5 \%$ for region 4 . Therefore, the baseline fit models for regions 2 and 3 do not include $p \pi^{-}$crossfeed (although it is taken into account as a part of the uncertainty due to modelling of nonresonant amplitudes), while for region 4 the $p \pi^{-}$nonresonant component is included in the model. Since only a small part of the $p \pi^{-}$helicity distribution enters the $D^{0} p$ fit region, the spin and parity assignment of the $p \pi^{-}$amplitude should have a very small effect. Thus only one partial wave $\left(J^{P}=1 / 2^{-}\right)$of the nonresonant $p \pi^{-}$component is included for the $D^{0} p$ amplitude fit.

\subsection{Fit in the region of $\Lambda_{c}(2880)^{+}$}

Next, an amplitude fit is performed in region 2, in the vicinity of the well-established $\Lambda_{c}(2880)^{+}$resonance. The quantum numbers of this state have been measured by the Belle collaboration to be $J^{P}=5 / 2^{+}[22,23]$. The fit probes the structure of the wide $D^{0} p$ 

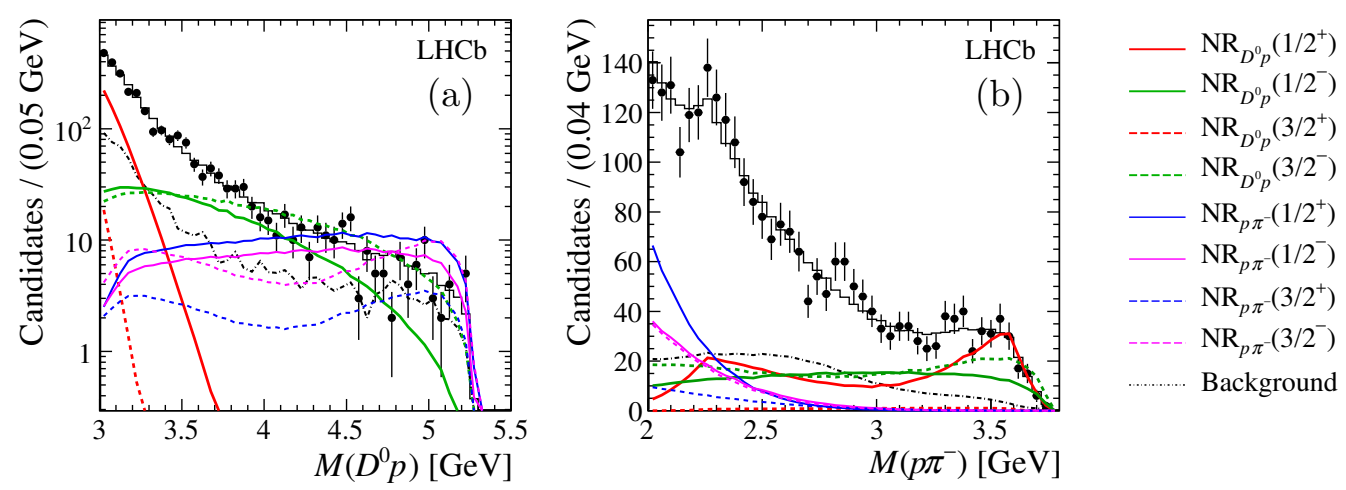

Figure 6. Fit results for the $\Lambda_{b}^{0} \rightarrow D^{0} p \pi^{-}$amplitude in the nonresonant region (region 1) (a) $M\left(D^{0} p\right)$ projection and (b) $M\left(p \pi^{-}\right)$projection. The points with error bars are data, the black histogram is the fit result, and coloured curves show the components of the fit model taking into account the efficiency. The dash-dotted line represents the background. Due to interference effects the total is not necessarily equal to the sum of the components.

\begin{tabular}{|c|cc|}
\hline Region & Signal yield & $p \pi^{-}$yield \\
\hline 2 & 1500 & 9 \\
3 & 2803 & 16 \\
4 & 4261 & 61 \\
\hline
\end{tabular}

Table 2. Estimated contributions from the $p \pi^{-}$nonresonant components in different phase space regions. The signal yields from table 1 are also included for comparison.

amplitude component underneath the $\Lambda_{c}(2880)^{+}$peak using the shape of the latter as a reference. Other $\Lambda_{c}(2880)^{+}$spin assignments from $1 / 2$ to $7 / 2$ are also tried (spin $7 / 2$ was not tested in the Belle analysis [22]). Since the amplitude is not sensitive to the absolute parities of the components, the parity of the $\Lambda_{c}(2880)^{+}$is always fixed to be positive; the parities of the other amplitude components are determined relative to its parity.

As for region 1, the nonresonant amplitude model consists of four contributions with spins $1 / 2$ and $3 / 2$ and both parities. The nonresonant components are parametrised either with the exponential model of eq. (3.13) ("Exponential"), or the amplitude with both real and imaginary parts varying linearly in $M^{2}\left(D^{0} p\right)$ ("Linear", which is a special case of the spline-interpolated shape with only two knots). The mass and width of the $\Lambda_{c}(2880)^{+}$ state are free parameters.

The model in which the $\Lambda_{c}(2880)^{+}$has spin $5 / 2$ is preferred for both nonresonant models, while the difference between exponential and linear models is negligible. The model with spin 5/2 and linear nonresonant amplitude parametrisation is taken as the baseline. Table 3 gives the differences in $\ln \mathcal{L}$ compared to the baseline, along with the $\chi^{2}$ values and the associated probabilities. The quality of the fit is obtained using the adaptive binning approach with at least 20 data entries in each bin and with the effective number of degrees of freedom ndf $\mathrm{eff}_{\text {f }}$ obtained from pseudoexperiments. The results of the fit with the baseline model are shown in figure 7 . 


\begin{tabular}{|l|c|rcc|}
\hline Nonresonant model & $\Lambda_{c}(2880)^{+} J^{P}$ & $\Delta \ln \mathcal{L}$ & $\chi^{2} / \mathrm{ndf}$ & $P\left(\chi^{2}, \mathrm{ndf}\right), \%$ \\
\hline \multirow{2}{*}{ Exponential } & $1 / 2^{+}$ & 41.5 & $108.9 / 70$ & 0.2 \\
& $3 / 2^{+}$ & 35.5 & $99.4 / 70$ & 1.2 \\
& $5 / 2^{+}$ & -0.2 & $65.6 / 70$ & 62.7 \\
& $7 / 2^{+}$ & 8.4 & $76.8 / 70$ & 27.0 \\
\hline \multirow{2}{*}{ Linear } & $1 / 2^{+}$ & 40.3 & $107.4 / 71$ & 0.3 \\
& $3 / 2^{+}$ & 35.7 & $98.8 / 71$ & 1.6 \\
& $\mathbf{5 / 2 ^ { + }}$ & $\mathbf{0 . 0}$ & $\mathbf{6 9 . 2} / \mathbf{7 1}$ & $\mathbf{5 3 . 8}$ \\
& $7 / 2^{+}$ & 8.6 & $76.2 / 71$ & 31.5 \\
\hline
\end{tabular}

Table 3. Values of the $\Delta \ln \mathcal{L}$ and fit quality for various $\Lambda_{c}(2880)^{+}$spin assignments and nonresonant amplitude models. The baseline model is shown in bold face.
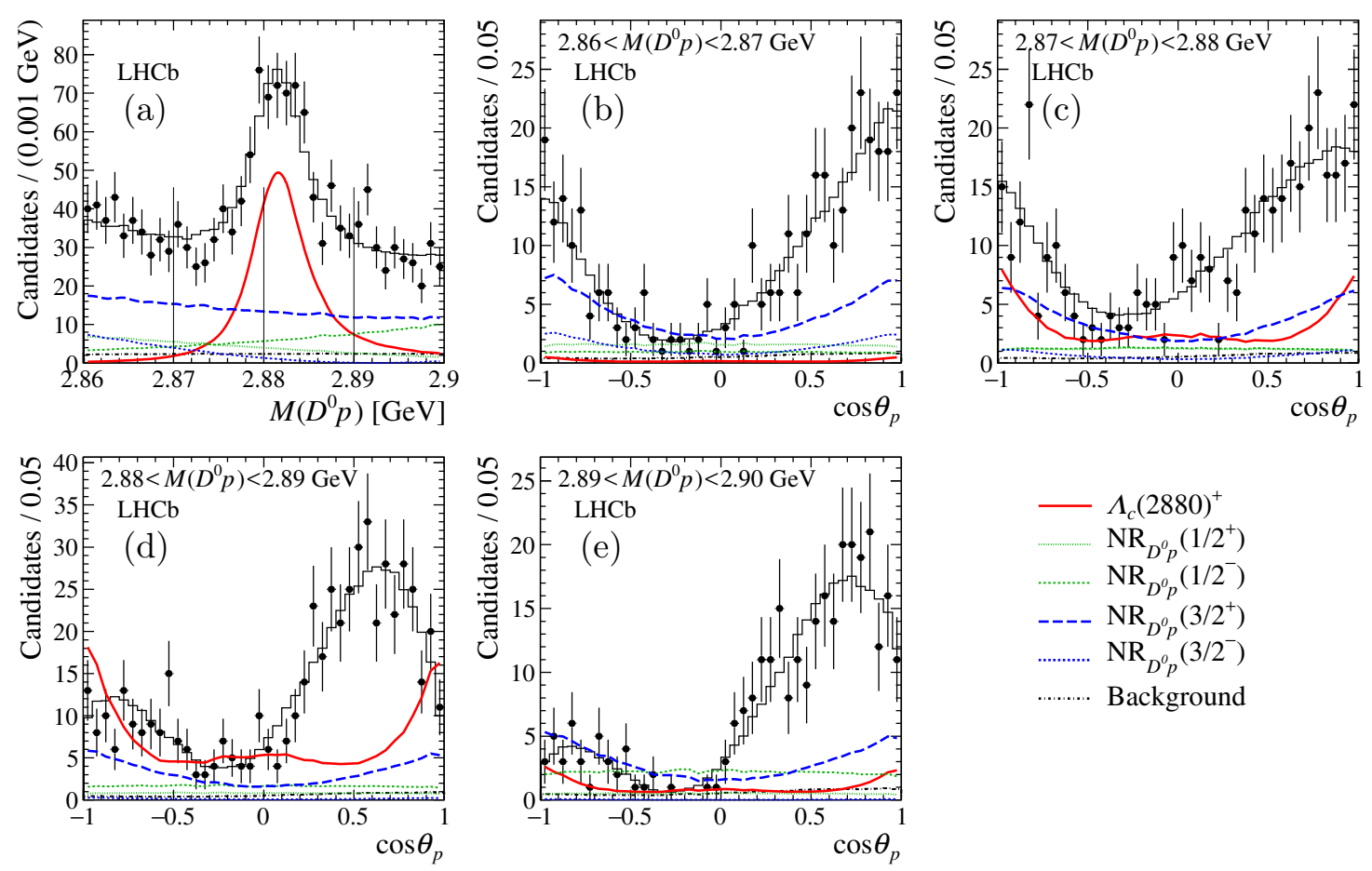

Figure 7. Results of the $\Lambda_{b}^{0} \rightarrow D^{0} p \pi^{-}$amplitude fit in the $\Lambda_{c}(2880)^{+}$mass region with spin-parity assignment $J^{P}=5 / 2^{+}$for the $\Lambda_{c}(2880)^{+}$resonance: (a) $M\left(D^{0} p\right)$ projection and (b-e) $\cos \theta_{p}$ projections in slices of the $D^{0} p$ invariant mass. The linear nonresonant model is used. Points with error bars are data, the black histogram is the fit result, coloured curves show the components of the fit model. The dash-dotted line represents the background. Vertical lines in (a) indicate the boundaries of the $D^{0} p$ invariant mass slices. Due to interference effects the total is not necessarily equal to the sum of the components. 

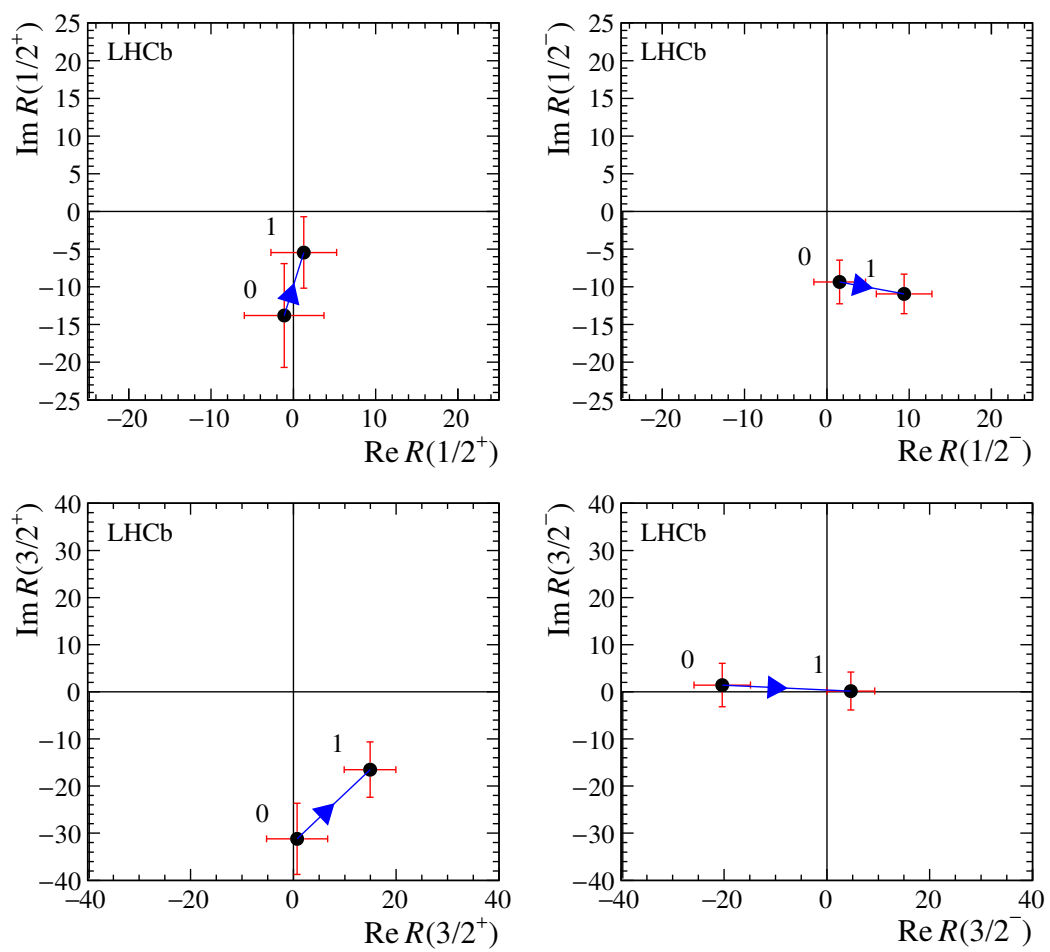

Figure 8. Argand diagrams for the four amplitude components underneath the $\Lambda_{c}(2880)^{+}$peak in the linear nonresonant model. In each diagram, point 0 corresponds to $M\left(D^{0} p\right)=2.86 \mathrm{GeV}$, and point 1 to $M\left(D^{0} p\right)=2.90 \mathrm{GeV}$.

Argand diagrams illustrating the amplitude and phase motion of the fit components are shown in figure 8 . The plots contain a hint of phase rotation for the $J^{P}=3 / 2^{+}$partial wave in a counter-clockwise direction, consistent with the resonance-like phase motion observed in the near-threshold fit (section 9.3). The statistical significance of this effect is studied with a series of pseudoexperiments where the samples are generated according to the fit where the complex phase in all the nonresonant components is constant. Each is fitted with two models, with the complex phase constrained to be the same for both endpoints, and floated freely. The distribution of the logarithmic likelihood difference $\Delta \ln \mathcal{L}$ between the two fits is studied and compared to the value obtained in data. The study shows that around $55 \%$ of the samples have $\Delta \ln \mathcal{L}$ greater than the value observed in data (1.4), i.e. this effect is not statistically significant with the data in region 2 alone.

Ensembles of pseudoexperiments, where the baseline model is used both to generate and to fit samples of the same size as in the data, are used to validate the statistical uncertainties obtained from the fit, check for systematic biases due to the fitting procedure, evaluate the statistical uncertainties on the fit fractions, and obtain the effective number of degrees of freedom for the fit quality evaluation based on a binned $\chi^{2}$ measure.

The unbinned maximum likelihood fit is unbiased only in the limit of a large data sample; in general a fit to a finite sample can exhibit a bias that is usually significantly smaller than the statistical uncertainty. Pseudoexperiments are used to evaluate and correct for 
such biases on the mass and the width of the $\Lambda_{c}(2880)^{+}$state, as well as on the fit fractions of the amplitude components obtained from the fit. The corrected values are

$$
\begin{aligned}
m\left(\Lambda_{c}(2880)^{+}\right) & =2881.75 \pm 0.29 \mathrm{MeV}, \\
\Gamma\left(\Lambda_{c}(2880)^{+}\right) & =5.43_{-0.71}^{+0.77} \mathrm{MeV} \\
\mathcal{F}\left(\Lambda_{c}(2880)^{+}\right) & =\left(29.0_{-3.6}^{+2.6}\right) \% \\
\mathcal{F}\left(1 / 2^{+}\right) & =\left(11.3_{-5.5}^{+2.2}\right) \% \\
\mathcal{F}\left(1 / 2^{-}\right) & =\left(16.3_{-2.6}^{+2.4}\right) \% \\
\mathcal{F}\left(3 / 2^{+}\right) & =\left(38.2_{-4.9}^{+5.0}\right) \% \\
\mathcal{F}\left(3 / 2^{-}\right) & =\left(7.8_{-3.1}^{+1.3}\right) \%
\end{aligned}
$$

The uncertainties are statistical only. Correlations between the fit parameters do not exceed $20 \%$. Since all the amplitude components have different quantum numbers, the interference terms cancel out after integrating over the phase space, and the sum of uncorrected fit fractions is exactly $100 \%$. After the bias correction is applied individually for each fit fraction, statistical fluctuations in the corrections lead to a small, statistically not significant, difference from $100 \%$ (in this case, the sum of fit fractions increases to $102.6 \%$ ).

A number of experimental systematic uncertainties on the $\Lambda_{c}(2880)^{+}$mass and width and on the difference $\Delta \ln \mathcal{L}$ between the baseline $(5 / 2)$ and the next-best $(7 / 2)$ spin assignments are considered and are given in table 4 . These arise from:

1. Uncertainty on the background fraction in the signal region (section 5). The statistical uncertainty is obtained from the fit to the $M\left(D^{0} p \pi^{-}\right)$distribution, and a systematic uncertainty arising from the modelling of the signal and background $M\left(D^{0} p \pi^{-}\right)$ distributions is estimated by performing fits with modified $M\left(D^{0} p \pi^{-}\right)$models. The sum in quadrature of these contributions is taken as the systematic uncertainty.

2. Uncertainty on the efficiency profile (section 6). The statistical uncertainty is evaluated via a bootstrapping procedure [56]. The uncertainty related to the kernel density estimation procedure is obtained by varying the kernel size. The uncertainty due to differences between data and simulation in the input variables of the BDT is estimated by varying the scaling factors for these variables. In addition, the replacement of simulated proton and pion PID variables with values drawn from control samples in the data with matching kinematics, described in section 4 , introduces further systematic uncertainties. The uncertainty associated with the limited size of these control samples is evaluated again with a bootstrapping procedure, and the uncertainty associated with the kinematic matching process is assessed by changing the kernel size in the nonparametric algorithm used to estimate the PID response as a function of the kinematic properties of the track.

3. Uncertainty on the background shape (section 7). This is assessed by varying the density estimation procedure (changing the number of Gaussian cores in the mixture model, or using kernel density estimation instead of a Gaussian mixture model), and by using only a narrower upper sideband of the $M\left(D^{0} p \pi^{-}\right)$distribution, 
$5680<M\left(D^{0} p \pi^{-}\right)<5780 \mathrm{MeV}$. The statistical uncertainty due to the finite size of the background sample is estimated by bootstrapping.

4. Uncertainty on the momentum resolution (section 8 ). This is estimated by varying the $M^{2}\left(D^{0} p\right)$ resolution by $15 \%$. It mainly affects the width of the $\Lambda_{c}(2880)^{+}$resonance.

5. Uncertainties on the mass scale. Due to the constraints on the hadron masses, the momentum scale uncertainty of the detector has a negligible effect on the fit. However, the uncertainties on the assigned mass values themselves do contribute. For $M\left(D^{0} p\right)$ amplitudes the dominant contribution comes from the $D^{0}$ mass uncertainty.

6. Uncertainty on the fit procedure itself. This is assessed by fitting ensembles of pseudoexperiments, where the baseline amplitude model is used for both generation and fitting, and the number of events generated for each pseudoexperiment is equal to the number of events in the data sample. The mean value for each fitted parameter is used as a correction for fitting bias, while the statistical uncertainty on the mean is taken as the uncertainty due to the fit procedure.

The uncertainties on the $D^{0}$ mass and the fit procedure do not affect the significance of the quantum number assignment and are thus not included in $\Delta \ln \mathcal{L}$ uncertainty.

Also reported in table 4 is the uncertainty related to the amplitude model. It consists of two contributions, corresponding to the uncertainties in the modelling of the resonant $\Lambda_{c}(2880)^{+}$shape and the nonresonant amplitudes. The model uncertainties are asymmetric, and the positive and negative uncertainties for the two components are combined in quadrature separately to obtain the total model uncertainty.

The uncertainty due to the Breit-Wigner parametrisation of the $\Lambda_{c}(2880)^{+}$amplitude is estimated by varying the radial parameters $r_{\Lambda_{b}^{0}}$ and $r_{\Lambda_{c}(2880)^{+}}$between 0 and $10 \mathrm{GeV}^{-1}$ and 0 and $3 \mathrm{GeV}^{-1}$, respectively, and by removing the angular barrier factor from the Breit-Wigner amplitude. The maximum deviation is taken as the uncertainty.

The uncertainty due to the modelling of the nonresonant amplitudes is estimated by taking the difference between the fit results obtained with the default linear nonresonant model and the alternative exponential model. The possible crossfeed from the $p \pi^{-}$channel is estimated by adding a $J^{P}=1 / 2^{-}$component in the $p \pi^{-}$channel to the amplitude. This component has a fixed exponential lineshape with shape parameter $\alpha=0.5 \mathrm{GeV}^{-2}$ (obtained in the fit to region 1 data) and its complex couplings are free parameters in the fit.

The helicity formalism used to describe the amplitudes is inherently non-relativistic. To assess the model uncertainty due to this limitation, an alternative description is obtained with covariant tensors using the qft++ framework [57], but it is much more expensive from a computational point of view and is therefore not used for the baseline fits. Differences between the helicity and the covariant formalism are mainly associated with the broad amplitude components and are therefore treated as a part of the uncertainty due to the nonresonant model. Although this contribution is included in the nonresonant model uncertainty in table 4 , it is also reported separately. 


\begin{tabular}{|l|ccc|}
\hline \multirow{2}{*}{ Source } & \multicolumn{3}{|c|}{ Uncertainty } \\
\cline { 2 - 4 } & $\begin{array}{c}m\left(\Lambda_{c}(2880)^{+}\right) \\
{[\mathrm{MeV}]}\end{array}$ & $\Gamma\left(\Lambda_{c}(2880)^{+}\right)$ & $\Delta \ln \mathcal{L}$ \\
\hline Background fraction & 0.01 & 0.02 & 0.11 \\
Efficiency profile & 0.01 & 0.10 & 0.35 \\
Background shape & 0.02 & 0.11 & 0.28 \\
Momentum resolution & 0.02 & 0.24 & 0.29 \\
Mass scale & 0.05 & - & - \\
Fit procedure & 0.03 & 0.08 & - \\
\hline Total systematic & 0.07 & 0.29 & 0.54 \\
\hline Breit-Wigner model & $+0.01 /-0.00$ & $+0.01 /-0.00$ & 0.01 \\
Nonresonant model & $+0.14 /-0.20$ & $+0.75 /-0.00$ & 0.62 \\
- of which helicity formalism & $+0.14 /-0.00$ & $+0.36 /-0.00$ & 0.62 \\
\hline Total model & $+0.14 /-0.20$ & $+0.75 /-0.00$ & 0.88 \\
\hline
\end{tabular}

Table 4. Systematic and model uncertainties on the $\Lambda_{c}(2880)^{+}$parameters and on the value of $\Delta \ln \mathcal{L}$ between the $5 / 2$ and $7 / 2$ spin assignments. The uncertainty due to the nonresonant model includes a component associated with the helicity formalism, which for comparison is given explicitly in the table, too.

The significance of the spin assignment $J=5 / 2$ with respect to the next most likely hypothesis $J=7 / 2$ for the $\Lambda_{c}(2880)^{+}$state is evaluated with a series of pseudoexperiments, where the samples are generated from the model with $J=7 / 2$ and then fitted with both $J=5 / 2$ and $7 / 2$ hypotheses. The difference of the logarithmic likelihoods $\Delta \ln \mathcal{L}$ is used as the test statistic. The distribution in $\Delta \ln \mathcal{L}$ is fitted with a Gaussian function and compared to the value of $\Delta \ln \mathcal{L}$ observed in data. The statistical significance is expressed in terms of a number of standard deviations $(\sigma)$. The uncertainty in $\Delta \ln \mathcal{L}$ due to systematic effects is small compared to the statistical uncertainty; combining them in quadrature results in an overall significance of $4.0 \sigma$. The fits with spins $1 / 2$ and $3 / 2$ for the $\Lambda_{c}(2880)^{+}$state yield large $\Delta \ln \mathcal{L}$ and poor fit quality, as seen from table 3 . These spin assignments are thus excluded.

In conclusion, the mass and width of the $\Lambda_{c}(2880)^{+}$resonance are found to be

$$
\begin{aligned}
m\left(\Lambda_{c}(2880)^{+}\right) & =2881.75 \pm 0.29(\text { stat }) \pm 0.07(\text { syst })_{-0.20}^{+0.14}(\text { model }) \mathrm{MeV} \\
\Gamma\left(\Lambda_{c}(2880)^{+}\right) & =5.43_{-0.71}^{+0.77}(\text { stat }) \pm 0.29(\text { syst })_{-0.00}^{+0.75}(\text { model }) \mathrm{MeV}
\end{aligned}
$$

These are consistent with the current world averages, and have comparable precision. The preferred value for the spin of this state is confirmed to be $5 / 2$, with a significance of $4 \sigma$ over the next most likely hypothesis, $7 / 2$. The spin assignments $1 / 2$ and $3 / 2$ are excluded. The largest nonresonant contribution underneath the $\Lambda_{c}(2880)^{+}$state comes from a partial wave with spin $3 / 2$ and positive parity. With a larger dataset, it would be possible to constrain the phase motion of the nonresonant amplitude in a model-independent way using the $\Lambda_{c}(2880)^{+}$amplitude as a reference. 


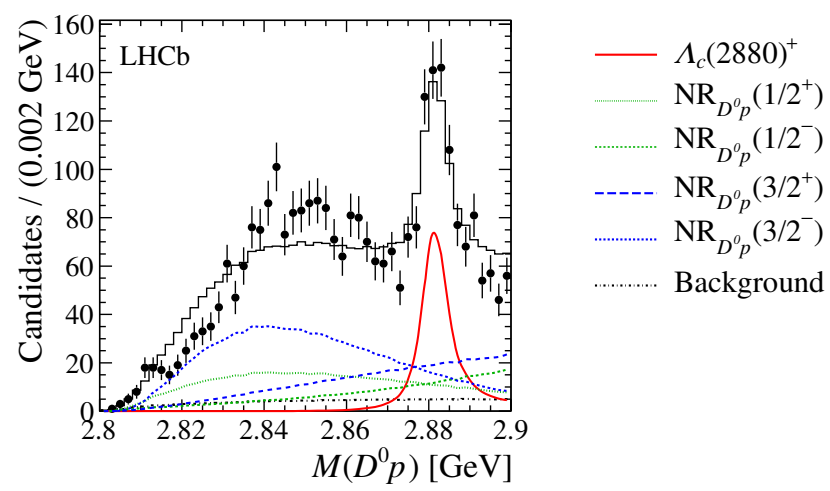

Figure 9. $M\left(D^{0} p\right)$ projections for the fit including the $\Lambda_{c}(2880)^{+}$state and four exponential nonresonant amplitudes.

\subsection{Fit in the near-threshold region}

Extending the $M\left(D^{0} p\right)$ range down to the $D^{0} p$ threshold (region 3), it becomes evident that a simple model for the broad amplitude components, such as an exponential lineshape, cannot describe the data (figure 9). The hypothesis that an additional resonance is present in the amplitude is tested in a model-dependent way by introducing a Breit-Wigner resonance in each of the $D^{0} p$ partial waves. Model-independent tests are also performed via fits in which one or more partial waves are parametrised with a spline-interpolated shape. The results of these tests are summarised in table 5 . The mass and width of the $\Lambda_{c}(2880)^{+}$ state are fixed to their known values [23] in these fits.

There are no states with mass around the $D^{0} p$ threshold $(2800 \mathrm{MeV})$ that are currently known to decay to the $D^{0} p$ final state. A broad structure has been seen previously in the $\Lambda_{c}^{+} \pi^{+} \pi^{-}$final state that is referred to as the $\Lambda_{c}(2765)^{+}$[58]. It could contribute to the $D^{0} p$ amplitude if its width is large. Since neither the quantum numbers nor the width of this structure have been measured, fits are carried out in which this structure is included, modelled as a Breit-Wigner amplitude with spin-parity $1 / 2^{ \pm}$or $3 / 2^{ \pm}$, and with a width that is free to vary; its mass is fixed to $2765 \mathrm{MeV}$. In addition, four exponential nonresonant components with $J^{P}=1 / 2^{+}, 1 / 2^{-}, 3 / 2^{+}$, and $3 / 2^{-}$are included. None of these fits are of acceptable quality, as shown in table 5. A Flatté parametrisation of the line shape [59] with couplings to $\Lambda_{c}^{+} \pi^{+} \pi^{-}$and $D^{0} p$ channels is also considered, but does not produce a fit of acceptable quality either. Therefore, a resonance with a fixed mass of $2765 \mathrm{MeV}$ is not sufficient to explain the data.

If the mass of the Breit-Wigner resonance is allowed to vary in the fit, good agreement with data can be obtained for the spin-parity assignment $J^{P}=3 / 2^{+}$. Moreover, if the resonance is assumed to have $J^{P}=3 / 2^{+}$, the exponential nonresonant component with $J^{P}=3 / 2^{+}$can be removed from the amplitude model without loss of fit quality. This model is taken as the baseline for this fit region. The mass and the width of the resonance obtained from the fit are around $2856 \mathrm{MeV}$ and $65 \mathrm{MeV}$, respectively, and therefore this structure will be referred to as $\Lambda_{c}(2860)^{+}$hereafter. The results of this fit are shown in figure 10 . 


\begin{tabular}{|c|c|c|c|c|c|c|c|c|}
\hline \multicolumn{4}{|c|}{ Nonresonant model } & \multicolumn{2}{|c|}{ Resonance } & \multirow[b]{2}{*}{$\Delta \ln \mathcal{L}$} & \multirow[b]{2}{*}{$\chi^{2} / \mathrm{ndf}$} & \multirow[b]{2}{*}{$P\left(\chi^{2}, \mathrm{ndf}\right)[\%]$} \\
\hline $1 / 2^{-}$ & $1 / 2^{+}$ & $3 / 2^{-}$ & $3 / 2^{+}$ & Mass $[\mathrm{MeV}]$ & $J^{P}$ & & & \\
\hline Exp & Exp & Exp & Exp & - & - & 72.2 & $287.4 / 150$ & 0.0 \\
\hline Exp & Exp & Exp & Exp & 2765 & $1 / 2^{-}$ & 53.6 & $247.2 / 146$ & 0.0 \\
\hline Exp & Exp & Exp & Exp & 2765 & $1 / 2^{+}$ & 52.8 & $254.8 / 146$ & 0.0 \\
\hline Exp & Exp & Exp & Exp & 2765 & $3 / 2^{-}$ & 45.8 & $240.5 / 146$ & 0.0 \\
\hline Exp & Exp & Exp & Exp & 2765 & $3 / 2^{+}$ & 38.5 & $226.0 / 146$ & 0.0 \\
\hline Exp & Exp & Exp & Exp & Float & $1 / 2^{-}$ & 8.2 & $162.7 / 145$ & 14.9 \\
\hline Exp & Exp & Exp & Exp & Float & $1 / 2^{+}$ & 15.2 & $170.2 / 145$ & 7.5 \\
\hline Exp & Exp & Exp & Exp & Float & $3 / 2^{-}$ & 9.3 & $162.1 / 145$ & 15.7 \\
\hline Exp & Exp & Exp & Exp & Float & $3 / 2^{+}$ & -3.3 & $139.5 / 145$ & 61.3 \\
\hline Exp & Exp & - & - & Float & $3 / 2^{+}$ & 12.8 & $169.7 / 153$ & 16.9 \\
\hline Exp & Exp & $\operatorname{Exp}$ & - & Float & $3 / 2^{+}$ & 0.0 & $143.1 / 149$ & 62.1 \\
\hline CSpl & Exp & Exp & Exp & - & - & 16.1 & $181.3 / 140$ & 1.1 \\
\hline Exp & CSpl & Exp & Exp & - & - & 2.0 & $154.8 / 140$ & 18.5 \\
\hline Exp & $\operatorname{Exp}$ & CSpl & Exp & - & - & 16.6 & $172.9 / 140$ & 3.1 \\
\hline Exp & Exp & Exp & CSpl & - & - & -0.4 & $146.6 / 140$ & 33.4 \\
\hline Exp & Exp & CSpl & - & - & - & 63.1 & $234.8 / 143$ & 0.0 \\
\hline Exp & Exp & - & CSpl & - & - & 10.8 & $165.7 / 143$ & 9.4 \\
\hline Exp & Exp & CSpl & $\mathrm{CSpl}$ & - & - & -4.7 & $146.1 / 130$ & 15.8 \\
\hline Exp & Exp & RSpl & Exp & - & - & 17.4 & 177.0/143 & 2.8 \\
\hline Exp & Exp & Exp & $\mathrm{RSpl}$ & - & - & 15.4 & $174.5 / 143$ & 3.8 \\
\hline Exp & Exp & RSpl & $\mathrm{RSpl}$ & - & - & -0.4 & $145.1 / 138$ & 32.3 \\
\hline
\end{tabular}

Table 5. Quality of various fits to the near-threshold $D^{0} p$ data. The models include nonresonant components for partial waves with $J \leq 3 / 2$ with or without a resonant component, whose mass is fixed to $2765 \mathrm{MeV}$ or allowed to vary ("Float"). "Exp" denotes an exponential nonresonant lineshape, "CSpl" a complex spline parametrisation, and "RSpl" a real spline parametrisation multiplied by a constant phase. The baseline model is shown in bold face.

One model-independent test for the presence of structure in the broad component is to describe the real and imaginary parts with spline-interpolated shapes. Cubic splines with six knots at $D^{0} p$ masses of $2800,2820,2840,2860,2880$ and $2900 \mathrm{MeV}$ are used. Of the models where only one partial wave is described by a spline while the others remain exponential, the best fit is again given by the model where the spline-interpolated amplitude has $J^{P}=3 / 2^{+}$. The Argand diagram for the $3 / 2^{+}$amplitude in this fit is shown in figure 11(a). Each of the points numbered from 0 to 5 corresponds to one spline knot at increasing values of $M\left(D^{0} p\right)$. Note that knots 3 and 5 at masses 2860 and $2900 \mathrm{MeV}$ correspond to the boundaries of the region 2 where the nonresonant amplitude is described by a linear function (section 9.1) and that the amplitudes and phases in those two knots can be compared directly to figure 8 , since the convention is the same in both fits. The Argand diagram demonstrates resonance-like phase rotation of the $3 / 2^{+}$partial wave with respect to the other broad components in the $D^{0} p$ amplitude, which are assumed to be constant in phase. Note that the absolute phase motion cannot be obtained from this fit since there are no reference amplitudes covering the entire $D^{0} p$ mass range used in the fit. 

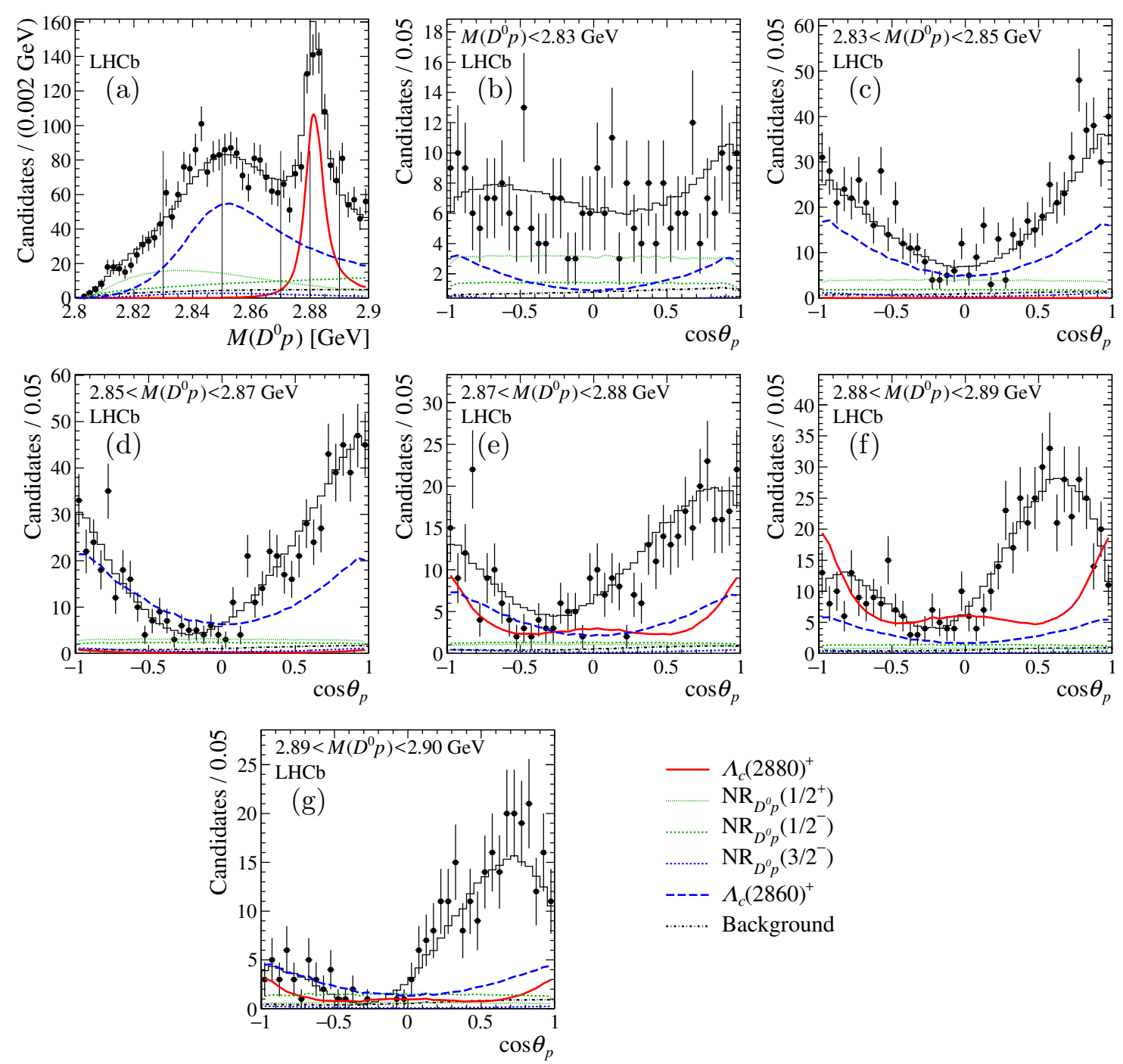

Figure 10. Results for the fit of the $\Lambda_{b}^{0} \rightarrow D^{0} p \pi^{-}$Dalitz plot distribution in the near-threshold $D^{0} p$ mass region (region 3 ): (a) $M\left(D^{0} p\right)$ projection, and (b-g) $\cos \theta_{p}$ projections for slices in $D^{0} p$ invariant mass. An exponential model is used for the nonresonant partial waves. A broad $\Lambda_{c}(2860)^{+}$ resonance and the $\Lambda_{c}(2880)^{+}$state are also present. Vertical lines in (a) indicate the boundaries of the $D^{0} p$ invariant mass slices. Due to interference effects the total is not necessarily equal to the sum of the components.

As seen in table 5 , inclusion of a spline-interpolated shape in the $1 / 2^{+}$component instead of $3 / 2^{+}$also gives a reasonable fit quality. The Argand diagram for the $1 / 2^{+}$wave in this fit is shown in figure 11(b). Since the phase rotates clockwise, this solution cannot be described by a single resonance.

A genuine resonance has characteristic phase motion as a function of $M\left(D^{0} p\right)$. As a null test, the fits are repeated with a spline function with no phase motion. This is implemented as a real spline function multiplied by a constant phase. The fits where only one partial wave is replaced by a real spline give poor fits. If both spin- $3 / 2$ amplitudes are 

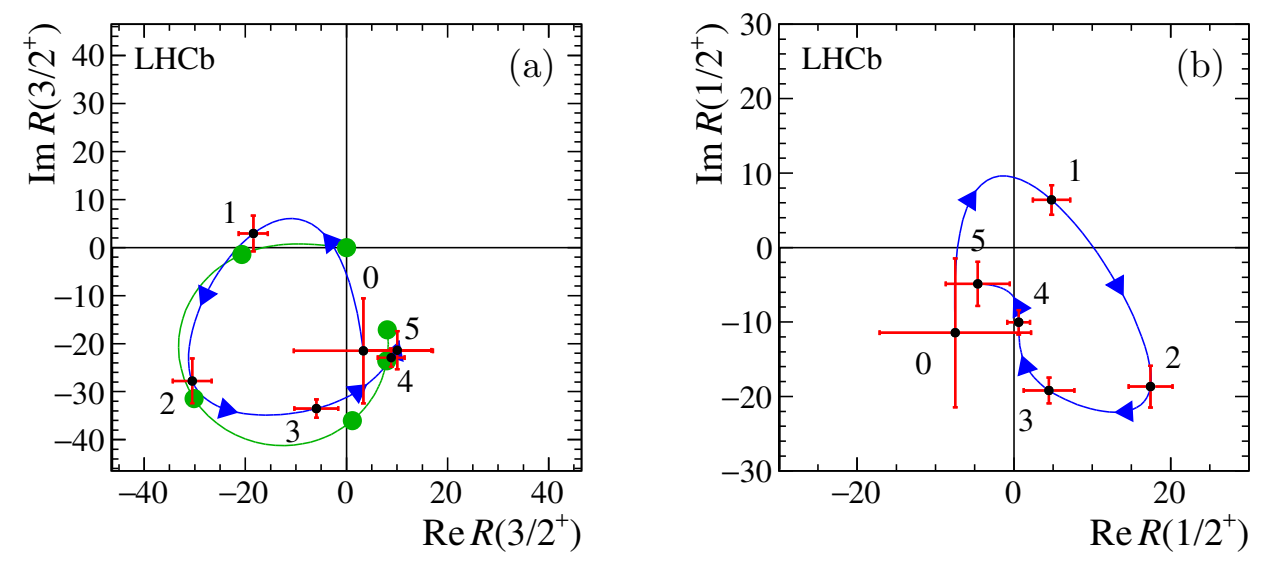

Figure 11. Argand diagrams for the complex spline components used in two fits, represented by blue lines with arrows indicating the phase motion with increasing $M\left(D^{0} p\right)$. For subfigure (a), the $J^{P}=3 / 2^{+}$partial wave is modelled as a spline and the other components in the fit $\left(1 / 2^{+}, 1 / 2^{-}\right.$ and $3 / 2^{-}$) are described with exponential amplitudes. For comparison, results from a separate fit in which the $3 / 2^{+}$partial wave is described with a Breit-Wigner function are superimposed: the green line represents its phase motion, and the green dots correspond to the $D^{0} p$ masses at the spline knots. For subfigure (b), the $J^{P}=1 / 2^{+}$component is modelled as a spline and $1 / 2^{-}, 3 / 2^{+}$ and $3 / 2^{-}$components as exponential amplitudes.
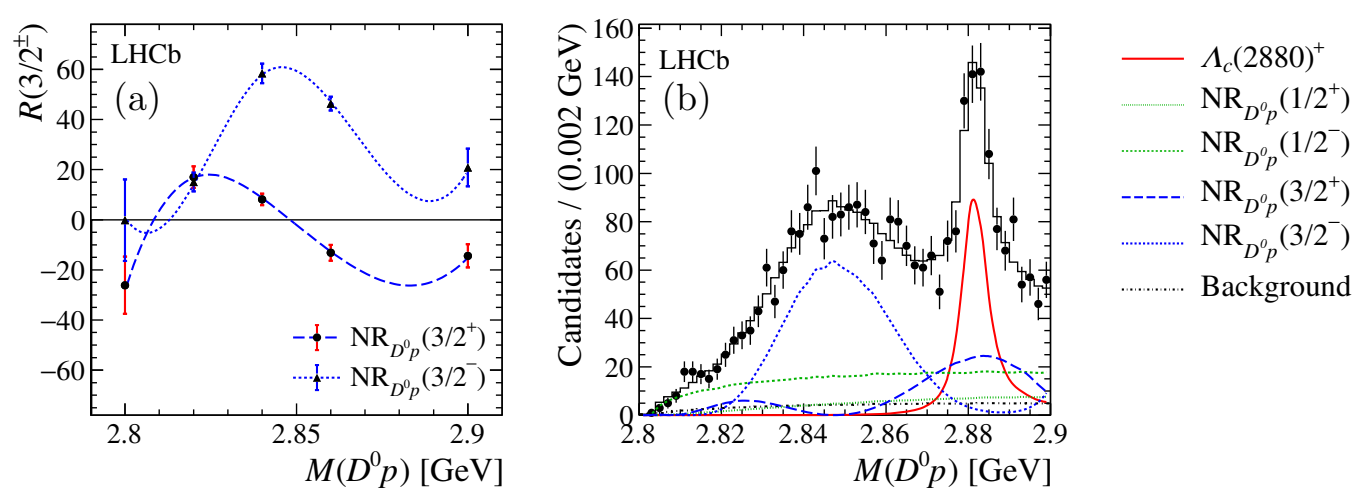

Figure 12. Results of the fit including the $\Lambda_{c}(2880)^{+}$state, two exponential nonresonant amplitudes with $J^{P}=1 / 2^{ \pm}$and two real splines in $J^{P}=3 / 2^{ \pm}$partial waves. (a) Spline amplitudes for $J^{P}=3 / 2^{ \pm}$partial waves as functions of $M\left(D^{0} p\right)$. Points with the error bars are fitted values of the amplitude in the spline knots, smooth curves are the interpolated amplitude shapes. (b) The $M\left(D^{0} p\right)$ projection of the decay density and the components of the fit model.

represented by real splines, the fit quality is good, but the resulting amplitudes oscillate as functions of $M\left(D^{0} p\right)$, which is not physical. Figure 12(a) shows the real spline amplitudes without the contribution of the phase space term, which exhibit oscillating behaviour, while figure $12(\mathrm{~b})$ shows the $M\left(D^{0} p\right)$ projection of the decay density for this solution.

As in the case of the amplitude fit in the $\Lambda_{c}(2880)^{+}$region, pseudoexperiments are used to validate the fit procedure, obtain uncertainties on the fit fractions, and deter- 
mine values of ndf $_{\text {eff }}$ for the binned fit quality test. Pseudoexperiments are also used to obtain the $\Delta \ln \mathcal{L}$ distributions for fits with various spin-parity hypotheses. After correcting for fit bias, the mass and width of the broad $\Lambda_{c}(2860)^{+}$resonance are found to be $m\left(\Lambda_{c}(2860)^{+}\right)=2856.1_{-1.7}^{+2.0} \mathrm{MeV}$ and $\Gamma\left(\Lambda_{c}(2860)^{+}\right)=67.6_{-8.1}^{+10.1} \mathrm{MeV}$, where the uncertainties are statistical only.

Systematic uncertainties are obtained following the same procedure as for the amplitude fit in the $\Lambda_{c}(2880)^{+}$region (section 9.2) and are summarised in table 6. An additional contribution to the list of systematic uncertainties is the uncertainty in the knowledge of the mass and width of the $\Lambda_{c}(2880)^{+}$resonance, which are fixed in the fit. It is estimated by varying these parameters within their uncertainties. The model uncertainty associated with the parametrisation of the nonresonant components is estimated by performing fits with an additional exponential $3 / 2^{+}$amplitude component and with the $3 / 2^{-}$component removed, as well as by adding the $p \pi^{-}$amplitude and using the covariant amplitude formalism in the same way as in section 9.2.

The $J^{P}=3 / 2^{+}$hypothesis is preferred for the $\Lambda_{c}(2860)^{+}$state, since its fit likelihood, as measured by $\Delta \ln \mathcal{L}$, is substantially better than those of the other $J^{P}$ values tested. The significance of this difference is assessed with pseudoexperiments and corresponds to $8.8 \sigma$, $6.3 \sigma$, and $6.6 \sigma$ for the $1 / 2^{+}, 1 / 2^{-}$, and $3 / 2^{-}$hypotheses, respectively. When systematic uncertainties are included, these reduce to $8.4 \sigma, 6.2 \sigma$ and $6.4 \sigma$. For $J^{P}=3 / 2^{+}$, the following parameters are obtained for the near-threshold resonant state:

$$
\begin{aligned}
m\left(\Lambda_{c}(2860)^{+}\right) & =2856.1_{-1.7}^{+2.0}(\text { stat }) \pm 0.5(\text { syst })_{-5.6}^{+1.1}(\text { model }) \mathrm{MeV} \\
\Gamma\left(\Lambda_{c}(2860)^{+}\right) & =67.6_{-8.1}^{+10.1}(\text { stat }) \pm 1.4(\text { syst })_{-20.0}^{+5.9}(\text { model }) \mathrm{MeV} .
\end{aligned}
$$

The largest uncertainties are associated with the modelling of the nonresonant components of the $D^{0} p$ amplitude.

\section{$9.4 \quad$ Fit including $\Lambda_{c}(2940)^{+}$}

Finally, the $D^{0} p$ mass region in the amplitude fit is extended up to $M\left(D^{0} p\right)=3.0 \mathrm{GeV}$ to include the $\Lambda_{c}(2940)^{+}$state (region 4 ). Since the behaviour of the slowly-varying $D^{0} p$ amplitude is consistent with the presence of a resonance in the $J^{P}=3 / 2^{+}$wave and nonresonant amplitudes in the $1 / 2^{+}, 1 / 2^{-}$, and $3 / 2^{-}$waves, the same model is used to describe those parts of the amplitude in the extended fit region. The $\Lambda_{c}(2940)^{+}$resonance is modelled by a Breit-Wigner lineshape. The masses and widths of the $\Lambda_{c}(2940)^{+}$and $\Lambda_{c}(2860)^{+}$states are floated in the fit, while those of the $\Lambda_{c}(2880)^{+}$resonance are fixed to their nominal values [23]. Several variants of the fit are performed in which the spin of $\Lambda_{c}(2940)^{+}$is assigned to be $1 / 2,3 / 2,5 / 2$ or $7 / 2$, with both positive and negative parities considered. Two different parametrisations of the nonresonant components are considered: the exponential model (taken as the baseline) and a second-order polynomial (eq. (3.14)).

The results of the fits are given in table 8 . For both nonresonant parametrisations, the best fit has a $\Lambda_{c}(2940)^{+}$spin-parity assignment of $3 / 2^{-}$. The results of the fit with this hypothesis and an exponential model for the nonresonant amplitudes, which is taken as the baseline for fit region 4 , are shown in figure 13. Although the $3 / 2^{-}$hypothesis describes 


\begin{tabular}{|c|c|c|c|c|c|}
\hline \multirow{3}{*}{ Source } & \multicolumn{5}{|c|}{ Uncertainty } \\
\hline & \multirow{2}{*}{$\begin{array}{c}m\left(\Lambda_{c}(2860)^{+}\right) \\
{[\mathrm{MeV}]}\end{array}$} & \multirow{2}{*}{$\begin{array}{c}\Gamma\left(\Lambda_{c}(2860)^{+}\right) \\
{[\mathrm{MeV}]}\end{array}$} & \multicolumn{3}{|c|}{$\Delta \ln \mathcal{L}$} \\
\hline & & & $1 / 2^{+}$ & $1 / 2^{-}$ & $3 / 2^{+}$ \\
\hline Background fraction & 0.22 & 0.54 & 2.3 & 1.1 & 1.8 \\
\hline Efficiency profile & 0.20 & 0.61 & 0.5 & 0.8 & 0.4 \\
\hline Background shape & 0.29 & 0.77 & 1.0 & 0.4 & 0.3 \\
\hline Momentum resolution & 0.10 & 0.49 & - & - & - \\
\hline Mass scale & 0.05 & - & - & - & - \\
\hline Fit procedure & 0.17 & 0.67 & - & - & - \\
\hline$\Lambda_{c}(2880)^{+}$parameters & 0.02 & 0.22 & 0.7 & 0.4 & 0.5 \\
\hline Total systematic & 0.46 & 1.41 & 2.7 & 1.4 & 2.0 \\
\hline Breit-Wigner model & $+1.11 /-1.65$ & $+5.92 /-8.02$ & 0.2 & 0.0 & 0.2 \\
\hline Nonresonant model & $+0.00 /-5.35$ & $+0.15 /-18.29$ & 2.4 & 0.1 & 0.5 \\
\hline — of which helicity formalism & $+0.00 /-1.23$ & $+0.00 /-5.67$ & 1.6 & 0.1 & 0.0 \\
\hline Total model & $+1.11 /-5.59$ & $+5.93 /-19.97$ & 2.9 & 0.2 & 0.5 \\
\hline
\end{tabular}

Table 6. Systematic uncertainties on the $\Lambda_{c}(2860)^{+}$parameters and on $\Delta \ln \mathcal{L}$ between the baseline $3 / 2^{+}$and alternative spin-parity assignments. The uncertainty due to the nonresonant model includes a component associated with the helicity formalism, which for comparison is given explicitly in the table, too.

the data significantly better than all others in fits using an exponential nonresonant model, this is not the case for the more flexible polynomial model: the assignment $J^{P}=5 / 2^{-}$is only slightly worse $(\Delta \ln \mathcal{L}=3.6)$ and a number of other spin-parity assignments are not excluded either.

In the baseline model, the mass of the $\Lambda_{c}(2940)^{+}$state is measured to be $m\left(\Lambda_{c}(2940)^{+}\right)=2944.8_{-2.5}^{+3.5} \mathrm{MeV}$, and the width is $\Gamma\left(\Lambda_{c}(2940)^{+}\right)=27.7_{-6.0}^{+8.2} \mathrm{MeV}$. The fit fractions for the resonant components of the $D^{0} p$ amplitude are $\mathcal{F}\left(\Lambda_{c}(2860)^{+}\right)=$ $\left(47.2_{-2.8}^{+2.9}\right) \%, \mathcal{F}\left(\Lambda_{c}(2880)^{+}\right)=\left(12.9_{-0.9}^{+1.0}\right) \%$, and $\mathcal{F}\left(\Lambda_{c}(2940)^{+}\right)=\left(8.2_{-1.1}^{+2.3}\right) \%$. All these uncertainties are statistical. Pseudoexperiments are used to correct for fit bias, which is small compared to the statistical uncertainties, and to determine the linear correlation coefficients for the statistical uncertainties between the measured masses, widths and fit fractions (table 7).

The systematic and model uncertainties for the parameters given above, obtained following the procedure described in sections 9.2 and 9.3, are presented in table 9. The part of the model uncertainty associated with the nonresonant amplitude is estimated from fits that use the polynomial nonresonant parametrisation instead of the default exponential form, by adding a $3 / 2^{+}$nonresonant amplitude or removing the $3 / 2^{-}$or $p \pi^{-}$amplitudes, and by using the covariant formalism instead of the baseline helicity formalism. The uncertainty due to the unknown quantum numbers of the $\Lambda_{c}(2940)^{+}$state is estimated from the variation among the fits with spin-parity assignments that give reasonable fit quality $\left(P\left(\chi^{2}\right.\right.$, ndf $\left.)>5 \%\right): 3 / 2^{+}, 3 / 2^{-}, 5 / 2^{+}, 5 / 2^{-}$. 


\begin{tabular}{|c|c|c|c|c|c|c|c|}
\hline & 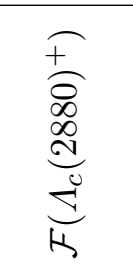 & 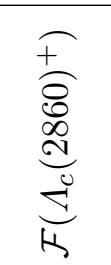 & 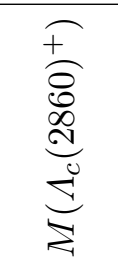 & 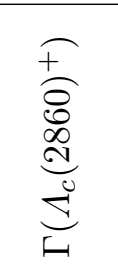 & 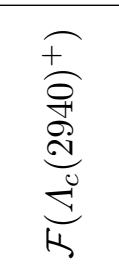 & 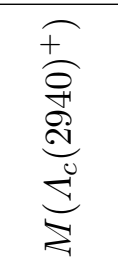 & 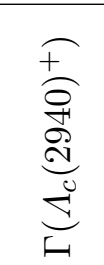 \\
\hline $\mathcal{F}\left(\Lambda_{c}(2880)^{+}\right)$ & +1.00 & & & & & & \\
\hline $\mathcal{F}\left(\Lambda_{c}(2860)^{+}\right)$ & +0.02 & +1.00 & & & & & \\
\hline$M\left(\Lambda_{c}(2860)^{+}\right)$ & -0.14 & +0.24 & +1.00 & & & & \\
\hline$\Gamma\left(\Lambda_{c}(2860)^{+}\right)$ & -0.14 & +0.34 & +0.61 & +1.00 & & & \\
\hline $\mathcal{F}\left(\Lambda_{c}(2940)^{+}\right)$ & +0.18 & +0.03 & -0.02 & -0.12 & +1.00 & & \\
\hline$M\left(\Lambda_{c}(2940)^{+}\right)$ & +0.02 & +0.13 & -0.08 & -0.09 & +0.45 & +1.00 & \\
\hline$\Gamma\left(\Lambda_{c}(2940)^{+}\right)$ & +0.15 & +0.06 & -0.04 & -0.11 & +0.78 & +0.54 & +1.00 \\
\hline
\end{tabular}

Table 7. Correlation matrix associated to the statistical uncertainties of the fit results in the fit region 4.

\begin{tabular}{|l|c|rcc|}
\hline Nonresonant model & $\Lambda_{c}(2940)^{+} J^{P}$ & $\Delta \ln \mathcal{L}$ & $\chi^{2} /$ ndf & $P\left(\chi^{2}\right.$, ndf $)[\%]$ \\
\hline Exponential & No $\Lambda_{c}^{+}(2940)$ & 54.6 & $337.3 / 230$ & 0.0 \\
& $1 / 2^{-}$ & 25.5 & $293.1 / 228$ & 0.2 \\
& $1 / 2^{+}$ & 34.2 & $306.4 / 228$ & 0.0 \\
& $\mathbf{3} \mathbf{2}^{-}$ & $\mathbf{0 . 0}$ & $\mathbf{2 4 6 . 9 / 2 2 8}$ & $\mathbf{1 8 . 6}$ \\
& $3 / 2^{+}$ & 14.8 & $269.1 / 228$ & 3.2 \\
& $5 / 2^{-}$ & 14.5 & $269.9 / 228$ & 3.0 \\
& $5 / 2^{+}$ & 15.6 & $271.7 / 228$ & 2.5 \\
& $7 / 2^{-}$ & 23.0 & $276.4 / 228$ & 1.6 \\
& $7 / 2^{+}$ & 29.0 & $300.2 / 228$ & 0.1 \\
\hline Polynomial & No $\Lambda_{c}^{+}(2940)$ & 25.5 & $296.0 / 228$ & 0.2 \\
& $1 / 2^{-}$ & 8.9 & $270.0 / 226$ & 2.4 \\
& $1 / 2^{+}$ & 7.2 & $266.1 / 226$ & 3.5 \\
& $3 / 2^{-}$ & -4.2 & $238.0 / 226$ & 27.9 \\
& $3 / 2^{+}$ & 4.9 & $253.4 / 226$ & 10.2 \\
& $5 / 2^{-}$ & -0.6 & $249.0 / 226$ & 14.0 \\
& $5 / 2^{+}$ & 4.9 & $250.5 / 226$ & 12.6 \\
& $7 / 2^{-}$ & 10.6 & $270.0 / 226$ & 2.4 \\
& $7 / 2^{+}$ & 11.7 & $273.0 / 226$ & 1.8 \\
\hline
\end{tabular}

Table 8. Fit quality for various $\Lambda_{c}(2940)^{+}$spin-parity assignments. Exponential and polynomial parametrisations of the nonresonant lineshapes are considered. The baseline model is shown in bold face. 

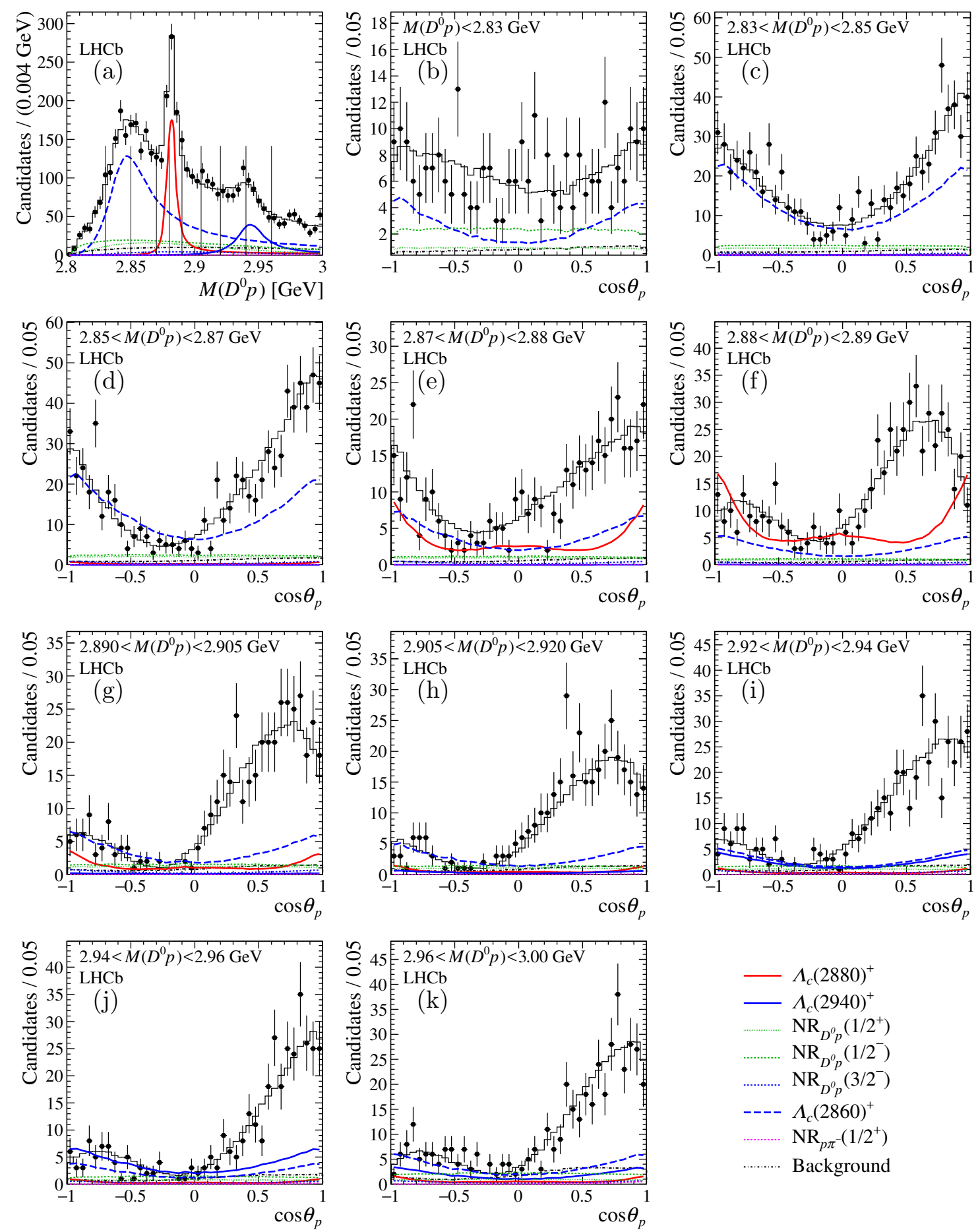

Figure 13. Results of the fit of the $\Lambda_{b}^{0} \rightarrow D^{0} p \pi^{-}$data in the $D^{0} p$ mass region including the $\Lambda_{c}(2880)^{+}$and $\Lambda_{c}(2940)^{+}$resonances (region 4$)$ : (a) $m\left(D^{0} p\right)$ projection and (b-k) $\cos \theta_{p}$ projections for slices of $D^{0} p$ invariant mass. An exponential model is used for the nonresonant partial waves, and the $J^{P}=3 / 2^{-}$hypothesis is used for the $\Lambda_{c}(2940)^{+}$state. Vertical lines in (a) indicate the boundaries of the $D^{0} p$ invariant mass slices. Due to interference effects the total is not necessarily equal to the sum of the components. 


\begin{tabular}{|l|ccccc|}
\hline \multirow{2}{*}{ Source } & \multicolumn{5}{|c|}{ Uncertainty } \\
\cline { 2 - 6 } & $\begin{array}{c}m\left(\Lambda_{c}(2940)^{+}\right) \\
{[\mathrm{MeV}]}\end{array}$ & $\Gamma\left(\Lambda_{c}(2940)^{+}\right)$ & $\mathcal{F}\left(\Lambda_{c}(2860)^{+}\right)$ & $\mathcal{F}\left(\Lambda_{c}(2880)^{+}\right)$ & $\mathcal{F}\left(\Lambda_{c}(2940)^{+}\right)$ \\
\hline Background fraction & 0.09 & 0.23 & 0.29 & 0.12 & 0.19 \\
Efficiency profile & 0.12 & 0.34 & 0.50 & 0.24 & 0.11 \\
Background shape & 0.15 & 0.68 & 1.13 & 0.09 & 0.48 \\
Momentum resolution & 0.07 & 0.09 & 0.03 & 0.07 & 0.02 \\
Mass scale & 0.05 & - & - & - & - \\
Fit procedure & 0.30 & 0.45 & 0.25 & 0.08 & 0.15 \\
$\Lambda_{c}(2880)^{+}$parameters & 0.01 & 0.16 & 0.17 & 0.03 & 0.03 \\
\hline Total systematic & 0.38 & 0.92 & 1.30 & 0.30 & 0.55 \\
\hline Breit-Wigner model & $+0.10 /-0.16$ & $+0.00 /-0.34$ & $+0.00 /-0.59$ & $+0.01 /-0.16$ & $+0.17 /-0.31$ \\
Nonresonant model & $+0.00 /-1.43$ & $+5.21 /-7.39$ & $+8.77 /-1.60$ & $+0.86 /-0.41$ & $+2.06 /-2.38$ \\
- of which hel. form. & $+0.00 /-0.38$ & $+2.18 /-0.00$ & $+1.15 /-0.00$ & $+0.00 /-0.23$ & $+0.38 /-0.00$ \\
$\Lambda_{c}(2940)^{+} J^{P}$ & $+0.00 /-4.32$ & $+0.00 /-7.25$ & $+0.00 /-5.79$ & $+0.00 /-0.67$ & $+0.00 /-3.29$ \\
\hline Total model & $+0.10 /-4.58$ & $+5.22 /-10.36$ & $+8.82 /-6.04$ & $+0.86 /-0.80$ & $+2.07 /-4.08$ \\
\hline
\end{tabular}

Table 9. Systematic and model uncertainties of the $\Lambda_{c}(2940)^{+}$parameters and the resonance fit fractions. The uncertainty due to the nonresonant model includes a component associated with the helicity formalism, which for comparison is given explicitly in the table, too.

The systematic uncertainties on $\Delta \ln \mathcal{L}$ between the various $\Lambda_{c}(2940)^{+}$spin-parity hypotheses and the baseline hypothesis, $J^{P}=3 / 2^{-}$, are shown in table 10 (for the exponential nonresonant model) and table 11 (for the polynomial model). Only those systematic variations from table 9 that can affect the significance of the quantum number assignment are considered. Since the cases with exponential and polynomial nonresonant amplitudes are treated separately, the model uncertainty associated with the nonresonant amplitudes does not include the difference between these two models.

For each $J^{P}$ hypothesis, the significance with respect to the baseline is obtained from ensembles of pseudoexperiments and shown in table 12. The column marked "Statistical" includes only statistical uncertainties on $\Delta \ln \mathcal{L}$, while that marked "Total" is the sum in quadrature of the statistical, systematic, and model uncertainties.

Including the systematic and model uncertainties, the mass and width of the $\Lambda_{c}(2940)^{+}$ resonance are

$$
\begin{aligned}
m\left(\Lambda_{c}(2940)^{+}\right) & =2944.8_{-2.5}^{+3.5}(\text { stat }) \pm 0.4(\text { syst })_{-4.6}^{+0.1}(\text { model }) \mathrm{MeV} \\
\Gamma\left(\Lambda_{c}(2940)^{+}\right) & =27.7_{-6.0}^{+8.2}(\text { stat }) \pm 0.9(\text { syst })_{-10.4}^{+5.2}(\text { model }) \mathrm{MeV}
\end{aligned}
$$

The largest uncertainties in the measurement of these parameters, apart from those of statistical origin, are related to the model of the nonresonant amplitude and the uncertainties for the $\Lambda_{c}(2940)^{+}$quantum numbers. The fit fractions of the resonances in the region of the $\Lambda_{b}^{0} \rightarrow D^{0} p \pi^{-}$phase space used in the fit, $M\left(D^{0} p\right)<3 \mathrm{GeV}$, are

$$
\begin{aligned}
& \mathcal{F}\left(\Lambda_{c}(2860)^{+}\right)=\left(47.2_{-2.8}^{+2.9}(\text { stat }) \pm 1.3(\text { syst })_{-6.0}^{+8.8}(\text { model })\right) \%, \\
& \mathcal{F}\left(\Lambda_{c}(2880)^{+}\right)=\left(12.9_{-0.9}^{+1.0}(\text { stat }) \pm 0.3(\text { syst })_{-0.8}^{+0.9}(\text { model })\right) \%, \\
& \mathcal{F}\left(\Lambda_{c}(2940)^{+}\right)=\left(8.2_{-1.1}^{+2.3}(\text { stat }) \pm 0.5(\text { syst })_{-4.1}^{+2.1}(\text { model })\right) \% .
\end{aligned}
$$




\begin{tabular}{|l|cccccccc|}
\hline & \multicolumn{7}{|c|}{$\Delta \ln \mathcal{L}$ uncertainty for $\Lambda_{c}(2940)^{+} J^{P}$} \\
\cline { 2 - 10 } Source & No $\Lambda_{c}(2940)^{+}$ & $1 / 2^{+}$ & $1 / 2^{-}$ & $3 / 2^{+}$ & $5 / 2^{+}$ & $5 / 2^{-}$ & $7 / 2^{+}$ & $7 / 2^{-}$ \\
\hline Background fraction & 0.3 & 0.7 & 0.3 & 0.9 & 0.7 & 0.6 & 0.7 & 0.8 \\
Efficiency profile & 0.3 & 0.2 & 0.6 & 0.6 & 0.6 & 0.6 & 0.9 & 1.1 \\
Background shape & 3.6 & 3.4 & 3.3 & 2.6 & 1.4 & 2.0 & 2.4 & 4.0 \\
Momentum resolution & 0.1 & 0.0 & 0.1 & 0.1 & 0.1 & 0.1 & 0.1 & 0.1 \\
$\Lambda_{c}(2880)^{+}$parameters & 0.2 & 0.2 & 0.9 & 0.2 & 0.3 & 0.1 & 0.5 & 0.4 \\
\hline Total systematic & 3.6 & 3.5 & 3.4 & 2.8 & 1.7 & 2.2 & 2.6 & 4.2 \\
\hline Breit-Wigner model & 2.1 & 1.2 & 1.9 & 1.6 & 2.3 & 0.4 & 1.4 & 1.4 \\
Nonresonant model & 3.7 & 2.4 & 0.4 & 1.5 & 1.0 & 1.9 & 1.4 & 0.1 \\
\hline Total model & 4.3 & 2.7 & 1.9 & 2.1 & 2.5 & 1.9 & 2.0 & 1.4 \\
\hline
\end{tabular}

Table 10. Systematic and model uncertainties on $\Delta \ln \mathcal{L}$ between the baseline fit with $J^{P}=3 / 2^{-}$ for the $\Lambda_{c}(2940)^{+}$state and other fits without a $\Lambda_{c}(2940)^{+}$contribution or with other spin-parity assignments, for the exponential nonresonant model.

\begin{tabular}{|l|cccccccc|}
\hline & \multicolumn{7}{|c|}{$\Delta \ln \mathcal{L}$ uncertainty for $\Lambda_{c}(2940)^{+} J^{P}$} \\
\cline { 2 - 10 } Source & No $\Lambda_{c}(2940)^{+}$ & $1 / 2^{+}$ & $1 / 2^{-}$ & $3 / 2^{+}$ & $5 / 2^{+}$ & $5 / 2^{-}$ & $7 / 2^{+}$ & $7 / 2^{-}$ \\
\hline Background fraction & 0.6 & 0.1 & 0.2 & 0.3 & 0.3 & 0.4 & 0.1 & 0.6 \\
Efficiency profile & 0.6 & 0.5 & 0.5 & 0.3 & 0.2 & 0.6 & 0.7 & 0.7 \\
Background shape & 1.2 & 0.5 & 0.6 & 1.4 & 1.6 & 0.7 & 1.5 & 1.3 \\
Momentum resolution & 0.5 & 0.2 & 0.1 & 0.1 & 0.1 & 0.1 & 0.1 & 0.1 \\
$\Lambda_{c}(2880)^{+}$parameters & 0.2 & 0.6 & 0.2 & 0.2 & 0.1 & 0.4 & 0.3 & 0.5 \\
\hline Total systematic & 1.6 & 0.9 & 0.8 & 1.5 & 1.6 & 1.1 & 1.7 & 1.7 \\
\hline Breit-Wigner model & 1.1 & 0.7 & 0.4 & 0.6 & 1.1 & 0.5 & 0.9 & 0.3 \\
Nonresonant model & 3.7 & 2.2 & 2.2 & 1.6 & 0.8 & 1.3 & 2.1 & 3.2 \\
\hline Total model & 3.8 & 2.3 & 2.3 & 1.7 & 1.3 & 1.4 & 2.3 & 3.2 \\
\hline
\end{tabular}

Table 11. Systematic and model uncertainties on $\Delta \ln \mathcal{L}$ between the baseline fit with $J^{P}=3 / 2^{-}$ for the $\Lambda_{c}(2940)^{+}$state and other fits without a $\Lambda_{c}(2940)^{+}$contribution or with other spin-parity assignments, for the polynomial nonresonant model.

The contributions of individual resonant components, integrated over the entire phase space of the $\Lambda_{b}^{0} \rightarrow D^{0} p \pi^{-}$decay, can be used to extract the ratios of branching fractions

$$
\begin{aligned}
& \left.\frac{\mathcal{B}\left(\Lambda_{b}^{0} \rightarrow \Lambda_{c}(2860)^{+} \pi^{-}\right) \times \mathcal{B}\left(\Lambda_{c}(2860)^{+} \rightarrow D^{0} p\right)}{\mathcal{B}\left(\Lambda_{b}^{0} \rightarrow \Lambda_{c}(2880)^{+} \pi^{-}\right) \times \mathcal{B}\left(\Lambda_{c}(2880)^{+} \rightarrow D^{0} p\right)}=4.54_{-0.39}^{+0.51} \text { (stat) } \pm 0.12 \text { (syst }\right)_{-0.58}^{+0.17} \text { (model) }, \\
& \left.\frac{\mathcal{B}\left(\Lambda_{b}^{0} \rightarrow \Lambda_{c}(2940)^{+} \pi^{-}\right) \times \mathcal{B}\left(\Lambda_{c}(2940)^{+} \rightarrow D^{0} p\right)}{\mathcal{B}\left(\Lambda_{b}^{0} \rightarrow \Lambda_{c}(2880)^{+} \pi^{-}\right) \times \mathcal{B}\left(\Lambda_{c}(2880)^{+} \rightarrow D^{0} p\right)}=0.83_{-0.10}^{+0.31} \text { (stat) } \pm 0.06 \text { (syst }\right)_{-0.43}^{+0.17} \text { (model) },
\end{aligned}
$$

which assumes the ratios of the branching fractions to be equal to the ratios of the fit fractions.

The constraints on the $\Lambda_{c}(2940)^{+}$quantum numbers depend on the description of the nonresonant amplitudes. If an exponential model is used for the nonresonant components, the single best spin-parity assignment is $J^{P}=3 / 2^{-}$, and the $3 / 2^{+}, 5 / 2^{+}$and $5 / 2^{-}$as- 


\begin{tabular}{|l|c|cc|}
\hline \multirow{2}{*}{ Nonresonant model } & $\Lambda_{c}(2940)^{+}$ & \multicolumn{2}{|c|}{ Significance, $\sigma$} \\
\cline { 3 - 4 } Exponential & $J^{P}$ & Statistical & Total \\
& No $\Lambda_{c}(2940)^{+}$ & 19.0 & 8.2 \\
& $1 / 2^{+}$ & 18.3 & 7.9 \\
& $1 / 2^{-}$ & 10.6 & 5.6 \\
& $3 / 2^{+}$ & 7.5 & 3.7 \\
& $5 / 2^{+}$ & 7.5 & 4.4 \\
& $5 / 2^{-}$ & 7.4 & 4.5 \\
& $7 / 2^{+}$ & 13.0 & 6.1 \\
& $7 / 2^{-}$ & 9.9 & 6.1 \\
\hline \multirow{3}{*}{ Polynomial } & No $\Lambda_{c}(2940)^{+}$ & 11.8 & 5.6 \\
& $1 / 2^{+}$ & 7.3 & 4.1 \\
& $1 / 2^{-}$ & 7.8 & 4.5 \\
& $3 / 2^{+}$ & 5.5 & 3.6 \\
& $5 / 2^{+}$ & 4.8 & 3.1 \\
& $5 / 2^{-}$ & 3.3 & 2.2 \\
& $7 / 2^{+}$ & 8.0 & 6.2 \\
& $7 / 2^{-}$ & 7.9 & 4.0 \\
\hline
\end{tabular}

Table 12. Significances of the $J^{P}=3 / 2^{-}$spin-parity assignment for $\Lambda_{c}(2940)^{+}$state with respect to the alternative models without a $\Lambda_{c}(2940)^{+}$contribution or with other spin-parity assignments.

signments are excluded at the levels of $3.7,4.4$ and 4.5 standard deviations, respectively (including systematic uncertainties), while spins of $1 / 2$ or $7 / 2$ are excluded by more than $5 \sigma$. If a polynomial nonresonant parametrisation is used, the solution with $3 / 2^{-}$is again the most likely one, though the data are consistent with the $5 / 2^{-}$hypothesis at $2.2 \sigma$. Several $J^{P}$ assignments $\left(5 / 2^{+}, 3 / 2^{+}, 7 / 2^{-}, 1 / 2^{+}\right.$and $\left.1 / 2^{-}\right)$are disfavoured with respect to the $3 / 2^{-}$hypothesis with significances between 3.1 and $4.5 \sigma$, and only the $7 / 2^{+}$hypothesis is excluded by more than $5 \sigma$. Since the data are consistent with both the exponential and polynomial nonresonant models, only weak constraints on the spin and parity are obtained, with $J^{P}=3 / 2^{-}$favoured and with positive parity excluded at the $3 \sigma$ level.

\section{Conclusion}

An amplitude analysis of the decay $\Lambda_{b}^{0} \rightarrow D^{0} p \pi^{-}$is performed in the region of the phase space containing $D^{0} p$ resonant contributions. This study provides important information about the structure of the $D^{0} p$ amplitude for future studies of CP violation in $\Lambda_{b}^{0} \rightarrow D p K^{-}$ decays, as well as on the spectroscopy of excited $\Lambda_{c}^{+}$states.

The preferred spin of the $\Lambda_{c}(2880)^{+}$state is found to be $J=5 / 2$, with the $J=7 / 2$ hypothesis disfavoured by 4.0 standard deviations. The solutions with $J=1 / 2$ and $3 / 2$ are excluded with a significance of more than 5 standard deviations. The mass and width 
of the $\Lambda_{c}(2880)^{+}$state are found to be:

$$
\begin{aligned}
m\left(\Lambda_{c}(2880)^{+}\right) & =2881.75 \pm 0.29(\text { stat }) \pm 0.07(\text { syst })_{-0.20}^{+0.14}(\text { model }) \mathrm{MeV} \\
\Gamma\left(\Lambda_{c}(2880)^{+}\right) & =5.43_{-0.71}^{+0.77}(\text { stat }) \pm 0.29(\text { syst })_{-0.00}^{+0.75}(\text { model }) \mathrm{MeV}
\end{aligned}
$$

These results are consistent with and have comparable precision to the current world averages (WA), which are $m_{\mathrm{WA}}\left(\Lambda_{c}(2880)^{+}\right)=2881.53 \pm 0.35 \mathrm{MeV}$, and $\Gamma_{\mathrm{WA}}\left(\Lambda_{c}(2880)^{+}\right)=$ $5.8 \pm 1.1 \mathrm{MeV}[23]$.

A near-threshold enhancement in the $D^{0} p$ amplitude is studied. The enhancement is consistent with being a resonant state (referred to here as the $\Lambda_{c}(2860)^{+}$) with mass and width

$$
\begin{aligned}
m\left(\Lambda_{c}(2860)^{+}\right) & =2856.1_{-1.7}^{+2.0}(\text { stat }) \pm 0.5(\text { syst })_{-5.6}^{+1.1}(\text { model }) \mathrm{MeV} \\
\Gamma\left(\Lambda_{c}(2860)^{+}\right) & =67.6_{-8.1}^{+10.1}(\text { stat }) \pm 1.4(\text { syst })_{-20.0}^{+5.9}(\text { model }) \mathrm{MeV}
\end{aligned}
$$

and quantum numbers $J^{P}=3 / 2^{+}$, with the parity measured relative to that of the $\Lambda_{c}(2880)^{+}$state. The other quantum numbers are excluded with a significance of more than 6 standard deviations. The phase motion of the $3 / 2^{+}$component with respect to the nonresonant amplitudes is obtained in a model-independent way and is consistent with resonant behaviour. With a larger dataset, it should be possible to constrain the phase motion of the $3 / 2^{+}$partial wave using the $\Lambda_{c}(2880)^{+}$amplitude as a reference, without making assumptions on the nonresonant amplitude behaviour. The mass of the $\Lambda_{c}(2860)^{+}$ state is consistent with recent predictions for an orbital $D$-wave $\Lambda_{c}^{+}$excitation with quantum numbers $3 / 2^{+}$based on the nonrelativistic heavy quark-light diquark model [24] and from QCD sum rules in the HQET framework [26].

First constraints on the spin and parity of the $\Lambda_{c}(2940)^{+}$state are obtained in this analysis, and its mass and width are measured. The most likely spin-parity assignment for $\Lambda_{c}(2940)^{+}$is $J^{P}=3 / 2^{-}$but the other solutions with spins $1 / 2$ to $7 / 2$ cannot be excluded. The mass and width of the $\Lambda_{c}(2940)^{+}$state are measured to be

$$
\begin{aligned}
m\left(\Lambda_{c}(2940)^{+}\right) & =2944.8_{-2.5}^{+3.5}(\text { stat }) \pm 0.4(\text { syst })_{-4.6}^{+0.1}(\text { model }) \mathrm{MeV} \\
\Gamma\left(\Lambda_{c}(2940)^{+}\right) & =27.7_{-6.0}^{+8.2}(\text { stat }) \pm 0.9(\text { syst })_{-10.4}^{+5.2}(\text { model }) \mathrm{MeV}
\end{aligned}
$$

The $J^{P}=3 / 2^{-}$assignment for $\Lambda_{c}(2940)^{+}$state is consistent with its interpretations as a $D^{*} N$ molecule $[16,17,19]$ or a radial $2 P$ excitation [21].

\section{Acknowledgments}

We express our gratitude to our colleagues in the CERN accelerator departments for the excellent performance of the LHC. We thank the technical and administrative staff at the LHCb institutes. We acknowledge support from CERN and from the national agencies: CAPES, CNPq, FAPERJ and FINEP (Brazil); NSFC (China); CNRS/IN2P3 (France); BMBF, DFG and MPG (Germany); INFN (Italy); FOM and NWO (The Netherlands); MNiSW and NCN (Poland); MEN/IFA (Romania); MinES and FASO (Russia); 
MinECo (Spain); SNSF and SER (Switzerland); NASU (Ukraine); STFC (United Kingdom); NSF (U.S.A.). We acknowledge the computing resources that are provided by CERN, IN2P3 (France), KIT and DESY (Germany), INFN (Italy), SURF (The Netherlands), PIC (Spain), GridPP (United Kingdom), RRCKI and Yandex LLC (Russia), CSCS (Switzerland), IFIN-HH (Romania), CBPF (Brazil), PL-GRID (Poland) and OSC (U.S.A.). We are indebted to the communities behind the multiple open source software packages on which we depend. Individual groups or members have received support from AvH Foundation (Germany), EPLANET, Marie Skłodowska-Curie Actions and ERC (European Union), Conseil Général de Haute-Savoie, Labex ENIGMASS and OCEVU, Région Auvergne (France), RFBR and Yandex LLC (Russia), GVA, XuntaGal and GENCAT (Spain), Herchel Smith Fund, The Royal Society, Royal Commission for the Exhibition of 1851 and the Leverhulme Trust (United Kingdom).

Open Access. This article is distributed under the terms of the Creative Commons Attribution License (CC-BY 4.0), which permits any use, distribution and reproduction in any medium, provided the original author(s) and source are credited.

\section{References}

[1] I. Dunietz, CP violation with beautiful baryons, Z. Phys. C 56 (1992) 129 [INSPIRE].

[2] Fayyazuddin, $\Lambda_{b}^{0} \rightarrow \Lambda+D^{0}\left(\bar{D}^{0}\right)$ decays and CP-violation, Mod. Phys. Lett. A 14 (1999) 63 [hep-ph/9806393] [INSPIRE].

[3] A.K. Giri, R. Mohanta and M.P. Khanna, Possibility of extracting the weak phase $\gamma$ from $\Lambda_{b}^{0} \rightarrow \Lambda D^{0}$ decays, Phys. Rev. D 65 (2002) 073029 [hep-ph/0112220] [inSPIRE].

[4] Y.K. Hsiao and C.Q. Geng, Direct CP-violation in $\Lambda_{b}$ decays, Phys. Rev. D 91 (2015) 116007 [arXiv: 1412.1899] [INSPIRE].

[5] W. Bensalem and D. London, T-violating triple-product correlations in hadronic b decays, Phys. Rev. D 64 (2001) 116003 [hep-ph/0005018] [INSPIRE].

[6] W. Bensalem, A. Datta and D. London, New-physics effects on triple-product correlations in $\Lambda_{b}^{0}$ decays, Phys. Rev. D 66 (2002) 094004 [hep-ph/0208054] [INSPIRE].

[7] W. Bensalem, A. Datta and D. London, T-violating triple-product correlations in charmless $\Lambda_{b}^{0}$ decays, Phys. Lett. B 538 (2002) 309 [hep-ph/0205009] [INSPIRE].

[8] LHCb collaboration, Observation of $J / \psi p$ resonances consistent with pentaquark states in $\Lambda_{b}^{0} \rightarrow J / \psi p K^{-}$decays, Phys. Rev. Lett. 115 (2015) 072001 [arXiv:1507.03414] [INSPIRE].

[9] LHCb collaboration, Studies of beauty baryon decays to $D^{0} p h^{-}$and $\Lambda_{c}^{+} h^{-}$final states, Phys. Rev. D 89 (2014) 032001 [arXiv:1311.4823] [INSPIRE].

[10] N. Cabibbo, Unitary symmetry and leptonic decays, Phys. Rev. Lett. 10 (1963) 531 [INSPIRE].

[11] M. Kobayashi and T. Maskawa, CP-violation in the renormalizable theory of weak interaction, Prog. Theor. Phys. 49 (1973) 652 [INSPIRE].

[12] Y. Dong, A. Faessler, T. Gutsche and V.E. Lyubovitskij, Strong two-body decays of the $\Lambda_{c}(2940)^{+}$in a hadronic molecule picture, Phys. Rev. D 81 (2010) 014006 [arXiv:0910.1204] [INSPIRE]. 
[13] Y. Dong, A. Faessler, T. Gutsche, S. Kumano and V.E. Lyubovitskij, Radiative decay of $\Lambda_{c}(2940)^{+}$in a hadronic molecule picture, Phys. Rev. D 82 (2010) 034035 [arXiv: 1006.4018] [INSPIRE].

[14] J. He, Y.-T. Ye, Z.-F. Sun and X. Liu, The observed charmed hadron $\Lambda_{c}(2940)^{+}$and the $D^{*} N$ interaction, Phys. Rev. D 82 (2010) 114029 [arXiv: 1008.1500] [InSPIRE].

[15] Y. Dong, A. Faessler, T. Gutsche, S. Kumano and V.E. Lyubovitskij, Strong three-body decays of $\Lambda_{c}(2940)^{+}$in a hadronic molecule picture, Phys. Rev. D 83 (2011) 094005 [arXiv: 1103.4762] [INSPIRE].

[16] J.-R. Zhang, $S$-wave $D^{(*)} N$ molecular states: $\Sigma_{c}(2800)$ and $\Lambda_{c}(2940)^{+}$?, Phys. Rev. D 89 (2014) 096006 [arXiv:1212.5325] [INSPIRE].

[17] P.G. Ortega, D.R. Entem and F. Fernández, Quark model description of the $\Lambda_{c}(2940)^{+}$as a molecular $D^{*} N$ state and the possible existence of the $\Lambda_{b}(6248)$, Phys. Lett. B 718 (2013) 1381 [arXiv: 1210.2633] [INSPIRE].

[18] Y. Dong, A. Faessler, T. Gutsche and V.E. Lyubovitskij, Role of the hadron molecule $\Lambda_{c}(2940)$ in the $p \bar{p} \rightarrow p D^{0} \bar{\Lambda}_{c}(2286)$ annihilation reaction, Phys. Rev. D 90 (2014) 094001 [arXiv: 1407.3949] [INSPIRE].

[19] J.-R. Zhang, Possibility of $\Sigma_{c}(2800)$ and $\Lambda_{c}(2940)^{+}$as $S$-wave $D^{(*)} N$ molecular states, Int. J. Mod. Phys. Conf. Ser. 29 (2014) 1460220 [arXiv:1405.0919] [InSPIRE].

[20] J.-J. Xie, Y.-B. Dong and X. Cao, Role of the $\Lambda_{c}(2940)^{+}$in the $\pi^{-} p \rightarrow D^{-} D^{0} p$ reaction close to threshold, Phys. Rev. D 92 (2015) 034029 [arXiv:1506.01133] [INSPIRE].

[21] B. Chen, K.-W. Wei and A. Zhang, Investigation of $\Lambda_{Q}$ and $\Xi_{Q}$ baryons in the heavy quark-light diquark picture, Eur. Phys. J. A 51 (2015) 82 [arXiv:1406.6561] [INSPIRE].

[22] Belle collaboration, K. Abe et al., Experimental constraints on the possible spin and parity of the $\Lambda_{c}(2880)^{+}$, Phys. Rev. Lett. 98 (2007) 262001 [hep-ex/0608043] [INSPIRE].

[23] Particle Data Group, C. Patrignani et al., Review of particle physics, Chin. Phys. C 40 (2016) 100001 [INSPIRE].

[24] B. Chen, K.-W. Wei, X. Liu and T. Matsuki, Low-lying charmed and charmed-strange baryon states, Eur. Phys. J. C 77 (2017) 154 [arXiv: 1609. 07967] [inSPIRE].

[25] Q.-F. Lü, Y. Dong, X. Liu and T. Matsuki, Puzzle of the $\Lambda_{c}$ spectrum, arXiv:1610.09605 [INSPIRE].

[26] H.-X. Chen, Q. Mao, A. Hosaka, X. Liu and S.-L. Zhu, D-wave charmed and bottomed baryons from QCD sum rules, Phys. Rev. D 94 (2016) 114016 [arXiv:1611.02677] [INSPIRE].

[27] W. Roberts and M. Pervin, Heavy baryons in a quark model, Int. J. Mod. Phys. A 23 (2008) 2817 [arXiv: 0711.2492] [INSPIRE].

[28] H.-Y. Cheng and C.-K. Chua, Strong decays of charmed baryons in heavy hadron chiral perturbation theory, Phys. Rev. D 75 (2007) 014006 [hep-ph/0610283] [INSPIRE].

[29] X.-H. Zhong and Q. Zhao, Charmed baryon strong decays in a chiral quark model, Phys. Rev. D 77 (2008) 074008 [arXiv:0711.4645] [INSPIRE].

[30] BABAR collaboration, B. Aubert et al., Observation of a charmed baryon decaying to $D^{0} p$ at a mass near $2.94 \mathrm{GeV} / c^{2}$, Phys. Rev. Lett. 98 (2007) 012001 [hep-ex/0603052] [INSPIRE].

[31] LHCb collaboration, The LHCb detector at the LHC, 2008 JINST 3 S08005 [INSPIRE]. 
[32] LHCb collaboration, LHCb detector performance, Int. J. Mod. Phys. A 30 (2015) 1530022 [arXiv: 1412.6352] [INSPIRE].

[33] R. Aaij et al., The LHCb trigger and its performance in 2011, 2013 JINST 8 P04022 [arXiv:1211.3055] [INSPIRE].

[34] V.V. Gligorov and M. Williams, Efficient, reliable and fast high-level triggering using a bonsai boosted decision tree, 2013 JINST 8 P02013 [arXiv:1210.6861] [INSPIRE].

[35] T. Sjöstrand, S. Mrenna and P.Z. Skands, PYTHIA 6.4 physics and manual, JHEP 05 (2006) 026 [hep-ph/0603175] [INSPIRE].

[36] T. Sjöstrand, S. Mrenna and P.Z. Skands, A brief introduction to PYTHIA 8.1, Comput. Phys. Commun. 178 (2008) 852 [arXiv:0710.3820] [INSPIRE].

[37] I. Belyaev et al., Handling of the generation of primary events in Gauss, the LHCb simulation framework, J. Phys. Conf. Ser. 331 (2011) 032047 [inSPIRE].

[38] D.J. Lange, The EvtGen particle decay simulation package, Nucl. Instrum. Meth. A 462 (2001) 152 [inSPIRE].

[39] P. Golonka and Z. Was, PHOTOS Monte Carlo: A precision tool for QED corrections in Z and $W$ decays, Eur. Phys. J. C 45 (2006) 97 [hep-ph/0506026] [INSPIRE].

[40] GEANT4 collaboration, J. Allison et al., Geant4 developments and applications, IEEE Trans. Nucl. Sci. 53 (2006) 270 [INSPIRE].

[41] GEANT4 collaboration, S. Agostinelli et al., GEANT4: A simulation toolkit, Nucl. Instrum. Meth. A 506 (2003) 250 [INSPIRE].

[42] M. Clemencic et al., The LHCb simulation application, Gauss: Design, evolution and experience, J. Phys. Conf. Ser. 331 (2011) 032023 [inSPIRE].

[43] LHCb collaboration, Amplitude analysis of $B^{+} \rightarrow J / \psi \phi K^{+}$decays, Phys. Rev. D 95 (2017) 012002 [arXiv: 1606.07898] [INSPIRE].

[44] LHCb collaboration, Evidence for exotic hadron contributions to $\Lambda_{b}^{0} \rightarrow J / \psi p \pi^{-}$decays, Phys. Rev. Lett. 117 (2016) 082003 [arXiv:1606. 06999] [INSPIRE].

[45] R.H. Dalitz, On the analysis of $\tau$-meson data and the nature of the $\tau$-meson, Phil. Mag. 44 (1953) 1068.

[46] LHCb collaboration, Measurements of the $\Lambda_{b}^{0} \rightarrow J / \psi \Lambda$ decay amplitudes and the $\Lambda_{b}^{0}$ polarisation in pp collisions at $\sqrt{s}=7 \mathrm{TeV}$, Phys. Lett. B 724 (2013) 27 [arXiv:1302.5578] [INSPIRE].

[47] J. Blatt and V.E. Weisskopf, Theoretical nuclear physics, J. Wiley, New York (1952).

[48] Belle collaboration, A. Garmash et al., Dalitz analysis of the three-body charmless decays $B^{+} \rightarrow K^{+} \pi^{+} \pi^{-}$and $B^{+} \rightarrow K^{+} K^{+} K^{-}$, Phys. Rev. D 71 (2005) 092003 [hep-ex/0412066] [INSPIRE].

[49] BaBAr collaboration, J.P. Lees et al., Study of CP-violation in Dalitz-plot analyses of $B^{0} \rightarrow K^{+} K^{-} K_{\mathrm{S}}^{0}, B^{+} \rightarrow K^{+} K^{-} K^{+}$and $B^{+} \rightarrow K_{\mathrm{S}}^{0} K_{\mathrm{S}}^{0} K^{+}$, Phys. Rev. D 85 (2012) 112010 [arXiv: 1201.5897] [INSPIRE].

[50] LHCb collaboration, Amplitude analysis of $B^{-} \rightarrow D^{+} \pi^{-} \pi^{-}$decays, Phys. Rev. D 94 (2016) 072001 [arXiv: 1608.01289] [INSPIRE]. 
[51] W.D. Hulsbergen, Decay chain fitting with a Kalman filter, Nucl. Instrum. Meth. A 552 (2005) 566 [physics/0503191] [INSPIRE].

[52] L. Breiman, J. H. Friedman, R. A. Olshen and C. J. Stone, Classification and regression trees, Wadsworth international group, Belmont, California, U.S.A. (1984).

[53] Y. Freund and R. E. Schapire, A decision-theoretic generalization of on-line learning and an application to boosting, J. Comput. Syst. Sci. 55 (1997) 119.

[54] A. Poluektov, Kernel density estimation of a multidimensional efficiency profile, 2015 JINST 10 P02011 [arXiv: 1411.5528] [INSPIRE].

[55] T. Skwarnicki, A study of the radiative CASCADE transitions between the Upsilon-Prime and Upsilon resonances, Ph.D. Thesis, Cracow, INP (1986).

[56] B. Efron, Bootstrap methods: Another look at the jackknife, Ann. Statist. 7 (1979) 1.

[57] M. Williams, Numerical object oriented quantum field theory calculations, Comput. Phys. Commun. 180 (2009) 1847 [arXiv:0805.2956] [INSPIRE].

[58] CLEO collaboration, M. Artuso et al., Observation of new states decaying into $\Lambda_{c}^{+} \pi^{-} \pi^{+}$, Phys. Rev. Lett. 86 (2001) 4479 [hep-ex/0010080] [INSPIRE].

[59] S.M. Flatté, Coupled-channel analysis of the $\pi \eta$ and $K \bar{K}$ systems near $K \bar{K}$ threshold, Phys. Lett. B 63 (1976) 224 [INSPIRE]. 


\section{The LHCb collaboration}

R. Aaij ${ }^{40}$, B. Adeva ${ }^{39}$, M. Adinolfi ${ }^{48}$, Z. Ajaltouni ${ }^{5}$, S. Akar ${ }^{59}$, J. Albrecht ${ }^{10}$, F. Alessio ${ }^{40}$, M. Alexander ${ }^{53}$, S. Ali ${ }^{43}$, G. Alkhazov ${ }^{31}$, P. Alvarez Cartelle ${ }^{55}$, A.A. Alves Jr ${ }^{59}$, S. Amato ${ }^{2}$, S. Amerio ${ }^{23}$, Y. Amhis ${ }^{7}$, L. An ${ }^{3}$, L. Anderlini ${ }^{18}$, G. Andreassi ${ }^{41}$, M. Andreotti ${ }^{17, g}$, J.E. Andrews ${ }^{60}$, R.B. Appleby ${ }^{56}$, F. Archilli ${ }^{43}$, P. d'Argent ${ }^{12}$, J. Arnau Romeu ${ }^{6}$, A. Artamonov ${ }^{37}$, M. Artuso ${ }^{61}$, E. Aslanides ${ }^{6}$, G. Auriemma ${ }^{26}$, M. Baalouch ${ }^{5}$, I. Babuschkin ${ }^{56}$, S. Bachmann ${ }^{12}$, J.J. Back ${ }^{50}$, A. Badalov ${ }^{38}$, C. Baesso ${ }^{62}$, S. Baker ${ }^{55}$, V. Balagura ${ }^{7, c}$, W. Baldini ${ }^{17}$, R.J. Barlow ${ }^{56}$, C. Barschel ${ }^{40}$, S. Barsuk ${ }^{7}$, W. Barter ${ }^{56}$, F. Baryshnikov ${ }^{32}$, M. Baszczyk ${ }^{27}$, V. Batozskaya ${ }^{29}$, B. Batsukh ${ }^{61}$, V. Battista ${ }^{41}$, A. Bay ${ }^{41}$, L. Beaucourt ${ }^{4}$, J. Beddow ${ }^{53}$, F. Bedeschi ${ }^{24}$, I. Bediaga ${ }^{1}$, A. Beiter ${ }^{61}$, L.J. Bel ${ }^{43}$, V. Bellee ${ }^{41}$, N. Belloli ${ }^{21, i}$, K. Belous ${ }^{37}$, I. Belyaev ${ }^{32}$, E. Ben-Haim ${ }^{8}$, G. Bencivenni ${ }^{19}$, S. Benson ${ }^{43}$, A. Berezhnoy ${ }^{33}$, R. Bernet ${ }^{42}$, A. Bertolin ${ }^{23}$, C. Betancourt ${ }^{42}$, F. Betti ${ }^{15}$, M.-O. Bettler ${ }^{40}$, M. van Beuzekom ${ }^{43}$, Ia. Bezshyiko ${ }^{42}$, S. Bifani ${ }^{47}$, P. Billoir ${ }^{8}$,

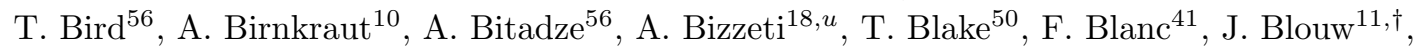
S. Blusk ${ }^{61}$, V. Bocci ${ }^{26}$, T. Boettcher ${ }^{58}$, A. Bondar ${ }^{36, w}$, N. Bondar ${ }^{31,40}$, W. Bonivento ${ }^{16}$, I. Bordyuzhin ${ }^{32}$, A. Borgheresi ${ }^{21, i}$, S. Borghi ${ }^{56}$, M. Borisyak ${ }^{35}$, M. Borsato ${ }^{39}$, F. Bossu ${ }^{7}$, M. Boubdir ${ }^{9}$, T.J.V. Bowcock ${ }^{54}$, E. Bowen ${ }^{42}$, C. Bozzi ${ }^{17,40}$, S. Braun ${ }^{12}$, M. Britsch ${ }^{12}$, T. Britton ${ }^{61}$, J. Brodzicka ${ }^{56}$, E. Buchanan ${ }^{48}$, C. Burr ${ }^{56}$, A. Bursche ${ }^{2}$, J. Buytaert ${ }^{40}$, S. Cadeddu ${ }^{16}$, R. Calabrese ${ }^{17, g}$, M. Calvi ${ }^{21, i}$, M. Calvo Gomez ${ }^{38, m}$, A. Camboni ${ }^{38}$, P. Campana ${ }^{19}$, D.H. Campora Perez ${ }^{40}$, L. Capriotti ${ }^{56}$, A. Carbone ${ }^{15, e}$, G. Carboni ${ }^{25, j}$, R. Cardinale ${ }^{20, h}$, A. Cardini ${ }^{16}$, P. Carniti ${ }^{21, i}$, L. Carson ${ }^{52}$, K. Carvalho Akiba ${ }^{2}$, G. Casse ${ }^{54}$, L. Cassina ${ }^{21, i}$, L. Castillo Garcia ${ }^{41}$, M. Cattaneo ${ }^{40}$, G. Cavallero ${ }^{20}$, R. Cenci ${ }^{24, t}$, D. Chamont ${ }^{7}$, M. Charles $^{8}$, Ph. Charpentier ${ }^{40}$, G. Chatzikonstantinidis ${ }^{47}$, M. Chefdeville ${ }^{4}$, S. Chen ${ }^{56}$, S.-F. Cheung ${ }^{57}$, V. Chobanova ${ }^{39}$, M. Chrzaszcz ${ }^{42,27}$, X. Cid Vidal ${ }^{39}$, G. Ciezarek $^{43}$, P.E.L. Clarke ${ }^{52}$,

M. Clemencic ${ }^{40}$, H.V. Cliff ${ }^{49}$, J. Closier ${ }^{40}$, V. Coco ${ }^{59}$, J. Cogan ${ }^{6}$, E. Cogneras ${ }^{5}$, V. Cogoni ${ }^{16,40, f}$, L. Cojocariu ${ }^{30}$, G. Collazuol ${ }^{23, o}$, P. Collins ${ }^{40}$, A. Comerma-Montells ${ }^{12}$, A. Contu ${ }^{40}$, A. Cook ${ }^{48}$, G. Coombs $^{40}$, S. Coquereau ${ }^{38}$, G. Corti ${ }^{40}$, M. Corvo ${ }^{17, g}$, C.M. Costa Sobral ${ }^{50}$, B. Couturier ${ }^{40}$, G.A. Cowan $^{52}$, D.C. Craik ${ }^{52}$, A. Crocombe ${ }^{50}$, M. Cruz Torres ${ }^{62}$, S. Cunliffe ${ }^{55}$, R. Currie ${ }^{55}$, C. D’Ambrosio ${ }^{40}$, F. Da Cunha Marinho ${ }^{2}$, E. Dall'Occo ${ }^{43}$, J. Dalseno ${ }^{48}$, P.N.Y. David ${ }^{43}$, A. Davis ${ }^{3}$, K. De Bruyn ${ }^{6}$, S. De Capua ${ }^{56}$, M. De Cian ${ }^{12}$, J.M. De Miranda ${ }^{1}$, L. De Paula ${ }^{2}$, M. De Serio ${ }^{14, d}$, P. De Simone ${ }^{19}$, C.T. Dean ${ }^{53}$, D. Decamp ${ }^{4}$, M. Deckenhoff ${ }^{10}$, L. Del Buono ${ }^{8}$, M. Demmer ${ }^{10}$, A. Dendek ${ }^{28}$, D. Derkach ${ }^{35}$, O. Deschamps ${ }^{5}$, F. Dettori ${ }^{40}$, B. Dey ${ }^{22}$, A. Di Canto ${ }^{40}$, H. Dijkstra ${ }^{40}$, F. Dordei ${ }^{40}$, M. Dorigo ${ }^{41}$, A. Dosil Suárez ${ }^{39}$, A. Dovbnya ${ }^{45}$, K. Dreimanis ${ }^{54}$, L. Dufour ${ }^{43}$, G. Dujany ${ }^{56}$, K. Dungs ${ }^{40}$, P. Durante ${ }^{40}$, R. Dzhelyadin ${ }^{37}$, A. Dziurda ${ }^{40}$, A. Dzyuba ${ }^{31}$, N. Déléage ${ }^{4}$, S. Easo ${ }^{51}$, M. Ebert ${ }^{52}$, U. Egede ${ }^{55}$, V. Egorychev ${ }^{32}$, S. Eidelman ${ }^{36, w}$, S. Eisenhardt ${ }^{52}$, U. Eitschberger ${ }^{10}$, R. Ekelhof ${ }^{10}$, L. Eklund ${ }^{53}$, S. Ely ${ }^{61}$, S. Esen ${ }^{12}$, H.M. Evans ${ }^{49}$, T. Evans ${ }^{57}$, A. Falabella ${ }^{15}$, N. Farley ${ }^{47}$, S. Farry ${ }^{54}$, R. Fay ${ }^{54}$, D. Fazzini ${ }^{21, i}$, D. Ferguson ${ }^{52}$, A. Fernandez Prieto ${ }^{39}$, F. Ferrari ${ }^{15,40}$, F. Ferreira Rodrigues ${ }^{2}$, M. Ferro-Luzzi ${ }^{40}$, S. Filippov ${ }^{34}$, R.A. Fini ${ }^{14}$, M. Fiore ${ }^{17, g}$, M. Fiorini ${ }^{17, g}$, M. Firlej ${ }^{28}$, C. Fitzpatrick ${ }^{41}$, T. Fiutowski ${ }^{28}$, F. Fleuret ${ }^{7, b}$, K. Fohl ${ }^{40}$, M. Fontana ${ }^{16,40}$, F. Fontanelli ${ }^{20, h}$, D.C. Forshaw ${ }^{61}$, R. Forty ${ }^{40}$, V. Franco Lima ${ }^{54}$, M. Frank ${ }^{40}$, C. Frei ${ }^{40}$, J. Fu ${ }^{22, q}$, W. Funk ${ }^{40}$, E. Furfaro ${ }^{25, j}$, C. Färber ${ }^{40}$, A. Gallas Torreira ${ }^{39}$, D. Galli ${ }^{15, e}$, S. Gallorini ${ }^{23}$, S. Gambetta ${ }^{52}$, M. Gandelman ${ }^{2}$, P. Gandini ${ }^{57}$, Y. Gao ${ }^{3}$, L.M. Garcia Martin ${ }^{69}$, J. García Pardiñas ${ }^{39}$, J. Garra Tico ${ }^{49}$, L. Garrido ${ }^{38}$, P.J. Garsed ${ }^{49}$, D. Gascon ${ }^{38}$, C. Gaspar ${ }^{40}$, L. Gavardi ${ }^{10}$, G. Gazzoni ${ }^{5}$, D. Gerick ${ }^{12}$, E. Gersabeck ${ }^{12}$, M. Gersabeck ${ }^{56}$, T. Gershon ${ }^{50}$, Ph. Ghez ${ }^{4}$, S. Gianì ${ }^{41}$, V. Gibson ${ }^{49}$, O.G. Girard ${ }^{41}$, L. Giubega ${ }^{30}$, K. Gizdov ${ }^{52}$, V.V. Gligorov ${ }^{8}$, D. Golubkov ${ }^{32}$, A. Golutvin ${ }^{55,40}$, A. Gomes ${ }^{1, a}$, I.V. Gorelov ${ }^{33}$, C. Gotti ${ }^{21, i}$, R. Graciani Diaz ${ }^{38}$, L.A. Granado Cardoso ${ }^{40}$, E. Graugés ${ }^{38}$, E. Graverini ${ }^{42}$, G. Graziani ${ }^{18}$, A. Grecu ${ }^{30}$, P. Griffith ${ }^{16}$, L. Grillo ${ }^{21,40, i}$, B.R. Gruberg Cazon ${ }^{57}$, O. Grünberg ${ }^{67}$, 
E. Gushchin ${ }^{34}$, Yu. Guz ${ }^{37}$, T. Gys ${ }^{40}$, C. Göbel ${ }^{62}$, T. Hadavizadeh ${ }^{57}$, C. Hadjivasiliou ${ }^{5}$, G. Haefeli ${ }^{41}$, C. Haen ${ }^{40}$, S.C. Haines ${ }^{49}$, B. Hamilton ${ }^{60}$, X. Han ${ }^{12}$, S. Hansmann-Menzemer ${ }^{12}$, N. Harnew ${ }^{57}$, S.T. Harnew ${ }^{48}$, J. Harrison ${ }^{56}$, M. Hatch ${ }^{40}$, J. He ${ }^{63}$, T. Head ${ }^{41}$, A. Heister ${ }^{9}$, K. Hennessy ${ }^{54}$, P. Henrard ${ }^{5}$, L. Henry ${ }^{8}$, E. van Herwijnen ${ }^{40}$, M. Heß ${ }^{67}$, A. Hicheur ${ }^{2}$, D. Hill ${ }^{57}$, C. Hombach ${ }^{56}$, H. Hopchev ${ }^{41}$, W. Hulsbergen ${ }^{43}$, T. Humair ${ }^{55}$, M. Hushchyn ${ }^{35}$, D. Hutchcroft ${ }^{54}$, M. Idzik ${ }^{28}$, P. Ilten ${ }^{58}$, R. Jacobsson ${ }^{40}$, A. Jaeger ${ }^{12}$, J. Jalocha ${ }^{57}$, E. Jans ${ }^{43}$, A. Jawahery ${ }^{60}$, F. Jiang ${ }^{3}$, M. John ${ }^{57}$, D. Johnson ${ }^{40}$, C.R. Jones ${ }^{49}$, C. Joram ${ }^{40}$, B. Jost ${ }^{40}$, N. Jurik ${ }^{57}$, S. Kandybei ${ }^{45}$, M. Karacson ${ }^{40}$, J.M. Kariuki ${ }^{48}$, S. Karodia ${ }^{53}$, M. Kecke ${ }^{12}$, M. Kelsey ${ }^{61}$, M. Kenzie ${ }^{49}$, T. Ketel ${ }^{44}$, E. Khairullin ${ }^{35}$, B. Khanji1 ${ }^{12}$, C. Khurewathanakull1, T. Kirn ${ }^{9}$, S. Klaver ${ }^{56}$, K. Klimaszewski ${ }^{29}$, S. Koliiev ${ }^{46}$, M. Kolpin ${ }^{12}$, I. Komarov ${ }^{41}$, R.F. Koopman ${ }^{44}$, P. Koppenburg ${ }^{43}$, A. Kosmyntseva ${ }^{32}$, A. Kozachuk ${ }^{33}$, M. Kozeiha ${ }^{5}$, L. Kravchuk ${ }^{34}$, K. Kreplin ${ }^{12}$, M. Kreps ${ }^{50}$, P. Krokovny ${ }^{36, w}$, F. Kruse ${ }^{10}$, W. Krzemien ${ }^{29}$, W. Kucewicz ${ }^{27, l}$, M. Kucharczyk ${ }^{27}$, V. Kudryavtsev ${ }^{36, w}$, A.K. Kuonen ${ }^{41}$, K. Kurek ${ }^{29}$, T. Kvaratskheliya ${ }^{32,40}$, D. Lacarrere ${ }^{40}$, G. Lafferty ${ }^{56}$, A. Lai ${ }^{16}$, G. Lanfranchi ${ }^{19}$, C. Langenbruch ${ }^{9}$, T. Latham ${ }^{50}$, C. Lazzeroni ${ }^{47}$, R. Le $\mathrm{Gac}^{6}$, J. van Leerdam ${ }^{43}$, A. Leflat ${ }^{33,40}$, J. Lefrançois ${ }^{7}$, R. Lefèvre ${ }^{5}$, F. Lemaitre ${ }^{40}$, E. Lemos $\mathrm{Cid}^{39}$, O. Leroy ${ }^{6}$, T. Lesiak ${ }^{27}$, B. Leverington ${ }^{12}$, T. $\mathrm{Li}^{3}$, Y. $\mathrm{Li}^{7}$, T. Likhomanenko ${ }^{35,68}$,

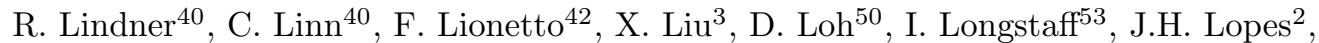
D. Lucchesi ${ }^{23, o}$, M. Lucio Martinez ${ }^{39}$, H. Luo ${ }^{52}$, A. Lupato ${ }^{23}$, E. Luppi ${ }^{17, g}$, O. Lupton ${ }^{40}$,

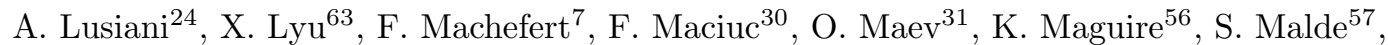
A. Malinin ${ }^{68}$, T. Maltsev ${ }^{36}$, G. Manca ${ }^{16, f}$, G. Mancinelli ${ }^{6}$, P. Manning ${ }^{61}$, J. Maratas $^{5, v}$, J.F. Marchand ${ }^{4}$, U. Marconi ${ }^{15}$, C. Marin Benito ${ }^{38}$, M. Marinangeli ${ }^{41}$, P. Marino ${ }^{24, t}$, J. Marks ${ }^{12}$, G. Martellotti ${ }^{26}$, M. Martin ${ }^{6}$, M. Martinelli ${ }^{41}$, D. Martinez Santos ${ }^{39}$, F. Martinez Vidal ${ }^{69}$, D. Martins Tostes ${ }^{2}$, L.M. Massacrier ${ }^{7}$, A. Massafferri ${ }^{1}$, R. Matev $^{40}$, A. Mathad ${ }^{50}$, Z. Mathe ${ }^{40}$, C. Matteuzzi ${ }^{21}$, A. Mauri ${ }^{42}$, E. Maurice ${ }^{7, b}$, B. Maurin ${ }^{41}$, A. Mazurov ${ }^{47}$, M. McCann ${ }^{55,40}$, A. $\mathrm{McNab}^{56}$, R. McNulty ${ }^{13}$, B. Meadows ${ }^{59}$, F. Meier ${ }^{10}$, M. Meissner ${ }^{12}$, D. Melnychuk ${ }^{29}$, M. Merk ${ }^{43}$, A. Merli², ${ }^{22,}$ E. Michielin ${ }^{23}$, D.A. Milanes ${ }^{66}$, M.-N. Minard ${ }^{4}$, D.S. Mitzel ${ }^{12}$, A. Mogini ${ }^{8}$, J. Molina Rodriguez ${ }^{1}$, I.A. Monroy ${ }^{66}$, S. Monteil ${ }^{5}$, M. Morandin ${ }^{23}$, P. Morawski ${ }^{28}$, A. Mordà ${ }^{6}$, M.J. Morello ${ }^{24, t}$, O. Morgunova ${ }^{68}$, J. Moron $^{28}$, A.B. Morris ${ }^{52}$, R. Mountain ${ }^{61}$, F. Muheim ${ }^{52}$, M. Mulder ${ }^{43}$, M. Mussini ${ }^{15}$, D. Müller ${ }^{56}$, J. Müller ${ }^{10}$, K. Müller ${ }^{42}$, V. Müller ${ }^{10}$, P. Naik ${ }^{48}$, T. Nakada ${ }^{41}$, R. Nandakumar ${ }^{51}$, A. Nandi ${ }^{57}$, I. Nasteva ${ }^{2}$, M. Needham ${ }^{52}$, N. Neri ${ }^{22}$, S. Neubert ${ }^{12}$, N. Neufeld ${ }^{40}$, M. Neuner ${ }^{12}$, T.D. Nguyen ${ }^{41}$, C. Nguyen-Mau ${ }^{41, n}$, S. Nieswand ${ }^{9}$, R. Niet ${ }^{10}$, N. Nikitin ${ }^{33}$, T. Nikodem ${ }^{12}$, A. Nogay ${ }^{68}$, A. Novoselov ${ }^{37}$, D.P. O'Hanlon ${ }^{50}$, A. Oblakowska-Mucha ${ }^{28}$, V. Obraztsov ${ }^{37}$, S. Ogilvy ${ }^{19}$, R. Oldeman ${ }^{16, f}$, C.J.G. Onderwater ${ }^{70}$, J.M. Otalora Goicochea ${ }^{2}$, A. Otto ${ }^{40}$, P. Owen ${ }^{42}$, A. Oyanguren ${ }^{69}$, P.R. Pais ${ }^{41}$, A. Palano ${ }^{14, d}$, M. Palutan ${ }^{19}$, A. Papanestis ${ }^{51}$, M. Pappagallo ${ }^{14, d}$, L.L. Pappalardo ${ }^{17, g}$, W. Parker ${ }^{60}$, C. Parkes ${ }^{56}$, G. Passaleva ${ }^{18}$, A. Pastore ${ }^{14, d}$, G.D. Patel ${ }^{54}$, M. Patel ${ }^{55}$, C. Patrignani ${ }^{15, e}$, A. Pearce ${ }^{40}$, A. Pellegrino ${ }^{43}$, G. Penso $^{26}$, M. Pepe Altarelli ${ }^{40}$, S. Perazzini ${ }^{40}$, P. Perret ${ }^{5}$, L. Pescatore ${ }^{41}$,

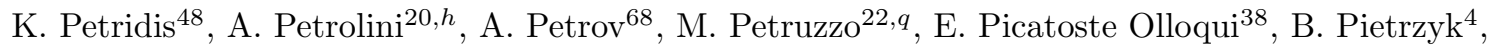
M. Pikies ${ }^{27}$, D. Pinci ${ }^{26}$, A. Pistone ${ }^{20}$, A. Piucci ${ }^{12}$, V. Placinta ${ }^{30}$, S. Playfer ${ }^{52}$, M. Plo Casasus ${ }^{39}$, T. Poikela ${ }^{40}$, F. Polci ${ }^{8}$, A. Poluektov ${ }^{50,36}$, I. Polyakov ${ }^{61}$, E. Polycarpo ${ }^{2}$, G.J. Pomery ${ }^{48}$, A. Popov $^{37}$, D. Popov ${ }^{11,40}$, B. Popovici ${ }^{30}$, S. Poslavskii ${ }^{37}$, C. Potterat ${ }^{2}$, E. Price ${ }^{48}$, J.D. Price ${ }^{54}$, J. Prisciandaro ${ }^{39,40}$, A. Pritchard ${ }^{54}$, C. Prouve ${ }^{48}$, V. Pugatch ${ }^{46}$, A. Puig Navarro ${ }^{42}$, G. Punzi ${ }^{24, p}$, W. Qian ${ }^{50}$, R. Quagliani ${ }^{7}, 48$, B. Rachwal ${ }^{27}$, J.H. Rademacker ${ }^{48}$, M. Rama ${ }^{24}$, M. Ramos Pernas ${ }^{39}$, M.S. Rangel ${ }^{2}$, I. Raniuk ${ }^{45}$, F. Ratnikov ${ }^{35}$, G. $\operatorname{Raven}^{44}$, F. Redi ${ }^{55}$, S. Reichert ${ }^{10}$, A.C. dos Reis ${ }^{1}$, C. Remon Alepuz ${ }^{69}$, V. Renaudin ${ }^{7}$, S. Ricciardi ${ }^{51}$, S. Richards ${ }^{48}$, M. Rihl ${ }^{40}$, K. Rinnert ${ }^{54}$, V. Rives Molina ${ }^{38}$, P. Robbe ${ }^{7,40}$, A.B. Rodrigues ${ }^{1}$, E. Rodrigues ${ }^{59}$, J.A. Rodriguez Lopez ${ }^{66}$, P. Rodriguez Perez ${ }^{56, \dagger}$, A. Rogozhnikov ${ }^{35}$, S. Roiser ${ }^{40}$, A. Rollings ${ }^{57}$, V. Romanovskiy ${ }^{37}$, A. Romero Vidal ${ }^{39}$, J.W. Ronayne ${ }^{13}$, M. Rotondo ${ }^{19}$, M.S. Rudolph ${ }^{61}$, T. Ruf ${ }^{40}$, P. Ruiz Valls ${ }^{69}$, 
J.J. Saborido Silva ${ }^{39}$, E. Sadykhov ${ }^{32}$, N. Sagidova ${ }^{31}$, B. Saitta ${ }^{16, f}$, V. Salustino Guimaraes ${ }^{1}$, C. Sanchez Mayordomo ${ }^{69}$, B. Sanmartin Sedes ${ }^{39}$, R. Santacesaria ${ }^{26}$, C. Santamarina Rios ${ }^{39}$, M. Santimaria ${ }^{19}$, E. Santovetti ${ }^{25, j}$, A. Sarti ${ }^{19, k}$, C. Satriano ${ }^{26, s}$, A. Satta ${ }^{25}$, D.M. Saunders ${ }^{48}$, D. Savrina ${ }^{32,33}$, S. Schael ${ }^{9}$, M. Schellenberg ${ }^{10}$, M. Schiller ${ }^{53}$, H. Schindler ${ }^{40}$, M. Schlupp ${ }^{10}$,

M. Schmelling ${ }^{11}$, T. Schmelzer ${ }^{10}$, B. Schmidt ${ }^{40}$, O. Schneider ${ }^{41}$, A. Schopper ${ }^{40}$, K. Schubert ${ }^{10}$, M. Schubiger ${ }^{41}$, M.-H. Schune ${ }^{7}$, R. Schwemmer ${ }^{40}$, B. Sciascia ${ }^{19}$, A. Sciubba ${ }^{26, k}$, A. Semennikov ${ }^{32}$, A. Sergi ${ }^{47}$, N. Serra ${ }^{42}$, J. Serrano ${ }^{6}$, L. Sestini ${ }^{23}$, P. Seyfert ${ }^{21}$, M. Shapkin ${ }^{37}$, I. Shapoval ${ }^{45}$, Y. Shcheglov ${ }^{31}$, T. Shears ${ }^{54}$, L. Shekhtman ${ }^{36, w}$, V. Shevchenko ${ }^{68}$, B.G. Siddi ${ }^{17,40}$,

R. Silva Coutinho ${ }^{42}$, L. Silva de Oliveira ${ }^{2}$, G. Simi $^{23, o}$, S. Simone ${ }^{14, d}$, M. Sirendi ${ }^{49}$, N. Skidmore ${ }^{48}$, T. Skwarnicki ${ }^{61}$, E. Smith ${ }^{55}$, I.T. Smith ${ }^{52}$, J. Smith ${ }^{49}$, M. Smith ${ }^{55}$, H. Snoek ${ }^{43}$, l. Soares Lavra ${ }^{1}$, M.D. Sokoloff ${ }^{59}$, F.J.P. Soler ${ }^{53}$, B. Souza De Paula ${ }^{2}$, B. Spaan ${ }^{10}$, P. $\operatorname{Spradlin}^{53}$, S. Sridharan ${ }^{40}$, F. Stagni ${ }^{40}$, M. Stahl ${ }^{12}$, S. Stahl ${ }^{40}$, P. Stefko ${ }^{41}$, S. Stefkova ${ }^{55}$, O. Steinkamp ${ }^{42}$, S. Stemmle ${ }^{12}$, O. Stenyakin ${ }^{37}$, H. Stevens ${ }^{10}$, S. Stevenson ${ }^{57}$, S. Stoica ${ }^{30}$, S. Stone ${ }^{61}$, B. Storaci ${ }^{42}$, S. Stracka ${ }^{24, p}$, M. Straticiuc ${ }^{30}$, U. Straumann ${ }^{42}$, L. Sun ${ }^{64}$, W. Sutcliffe ${ }^{55}$, K. Swientek ${ }^{28}$, V. Syropoulos ${ }^{44}$,

M. Szczekowski ${ }^{29}$, T. Szumlak ${ }^{28}$, S. T'Jampens ${ }^{4}$, A. Tayduganov ${ }^{6}$, T. Tekampe ${ }^{10}$, G. Tellarini ${ }^{17, g}$, F. Teubert ${ }^{40}$, E. Thomas ${ }^{40}$, J. van Tilburg ${ }^{43}$, M.J. Tilley ${ }^{55}$, V. Tisserand ${ }^{4}$, M. Tobin ${ }^{41}$, S. Tolk ${ }^{49}$, L. Tomassetti ${ }^{17, g}$, D. Tonelli ${ }^{40}$, S. Topp-Joergensen ${ }^{57}$, F. Toriello ${ }^{61}$, E. Tournefier ${ }^{4}$, S. Tourneur ${ }^{41}$,

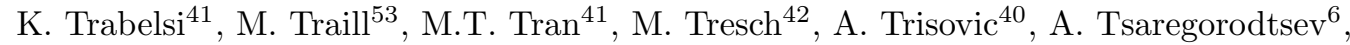
P. Tsopelas ${ }^{43}$, A. Tully ${ }^{49}$, N. Tuning ${ }^{43}$, A. Ukleja ${ }^{29}$, A. Ustyuzhanin ${ }^{35}$, U. Uwer ${ }^{12}$, C. Vacca ${ }^{16, f}$, V. Vagnoni ${ }^{15,40}$, A. Valassi ${ }^{40}$, S. Valat ${ }^{40}$, G. Valenti ${ }^{15}$, R. Vazquez Gomez ${ }^{19}$,

P. Vazquez Regueiro ${ }^{39}$, S. Vecchi ${ }^{17}$, M. van Veghel ${ }^{43}$, J.J. Velthuis ${ }^{48}$, M. Veltri ${ }^{18, r}$, G. Veneziano ${ }^{57}$, A. Venkateswaran ${ }^{61}$, M. Vernet ${ }^{5}$, M. Vesterinen ${ }^{12}$, J.V. Viana Barbosa ${ }^{40}$, B. Viaud ${ }^{7}$, D. Vieira ${ }^{63}$, M. Vieites Diaz ${ }^{39}$, H. Viemann ${ }^{67}$, X. Vilasis-Cardona ${ }^{38, m}$, M. Vitti ${ }^{49}$, V. Volkov ${ }^{33}$, A. Vollhardt ${ }^{42}$, B. Voneki ${ }^{40}$, A. Vorobyev ${ }^{31}$, V. Vorobyev ${ }^{36, w}$, C. Voß ${ }^{9}$, J.A. de Vries ${ }^{43}$, C. Vázquez Sierra ${ }^{39}$, R. Waldi ${ }^{67}$, C. Wallace ${ }^{50}$, R. Wallace ${ }^{13}$, J. Walsh ${ }^{24}$, J. Wang ${ }^{61}$, D.R. Ward ${ }^{49}$, H.M. Wark ${ }^{54}$, N.K. Watson ${ }^{47}$, D. Websdale ${ }^{55}$, A. Weiden ${ }^{42}$, M. Whitehead ${ }^{40}$, J. Wicht ${ }^{50}$, G. Wilkinson ${ }^{57,40}$, M. Wilkinson ${ }^{61}$, M. Williams ${ }^{40}$, M.P. Williams ${ }^{47}$, M. Williams ${ }^{58}$, T. Williams ${ }^{47}$, F.F. Wilson ${ }^{51}$, J. Wimberley ${ }^{60}$, J. Wishahi ${ }^{10}$, W. Wislicki ${ }^{29}$, M. Witek ${ }^{27}$, G. Wormser ${ }^{7}$, S.A. Wotton ${ }^{49}$, K. Wraight ${ }^{53}$, K. Wyllie ${ }^{40}$, Y. Xie ${ }^{65}$, Z. Xing ${ }^{61}$, Z. Xu ${ }^{4}$, Z. Yang ${ }^{3}$, Y. Yao ${ }^{61}$, H. Yin ${ }^{65}$, J. Yu ${ }^{65}$, X. Yuan ${ }^{36, w}$, O. Yushchenko ${ }^{37}$, K.A. Zarebski ${ }^{47}$, M. Zavertyaev ${ }^{11, c}$, L. Zhang ${ }^{3}$, Y. Zhang ${ }^{7}$, Y. Zhang ${ }^{63}$, A. Zhelezov ${ }^{12}$, Y. Zheng ${ }^{63}$, X. Zhu ${ }^{3}$, V. Zhukov ${ }^{33}$, S. Zucchelli ${ }^{15}$

1 Centro Brasileiro de Pesquisas Físicas (CBPF), Rio de Janeiro, Brazil

2 Universidade Federal do Rio de Janeiro (UFRJ), Rio de Janeiro, Brazil

3 Center for High Energy Physics, Tsinghua University, Beijing, China

4 LAPP, Université Savoie Mont-Blanc, CNRS/IN2P3, Annecy-Le-Vieux, France

5 Clermont Université, Université Blaise Pascal, CNRS/IN2P3, LPC, Clermont-Ferrand, France

6 CPPM, Aix-Marseille Université, CNRS/IN2P3, Marseille, France

7 LAL, Université Paris-Sud, CNRS/IN2P3, Orsay, France

8 LPNHE, Université Pierre et Marie Curie, Université Paris Diderot, CNRS/IN2P3, Paris, France

9 I. Physikalisches Institut, RWTH Aachen University, Aachen, Germany

10 Fakultät Physik, Technische Universität Dortmund, Dortmund, Germany

11 Max-Planck-Institut für Kernphysik (MPIK), Heidelberg, Germany

12 Physikalisches Institut, Ruprecht-Karls-Universität Heidelberg, Heidelberg, Germany

13 School of Physics, University College Dublin, Dublin, Ireland

14 Sezione INFN di Bari, Bari, Italy

15 Sezione INFN di Bologna, Bologna, Italy

16 Sezione INFN di Cagliari, Cagliari, Italy

17 Sezione INFN di Ferrara, Ferrara, Italy

18 Sezione INFN di Firenze, Firenze, Italy 
Sezione INFN di Milano Bicocca, Milano, Italy

22 Sezione INFN di Milano, Milano, Italy

23 Sezione INFN di Padova, Padova, Italy

24 Sezione INFN di Pisa, Pisa, Italy

25 Sezione INFN di Roma Tor Vergata, Roma, Italy

26 Sezione INFN di Roma La Sapienza, Roma, Italy

27 Henryk Niewodniczanski Institute of Nuclear Physics Polish Academy of Sciences, Kraków, Poland

28 AGH - University of Science and Technology, Faculty of Physics and Applied Computer Science, Kraków, Poland

29 National Center for Nuclear Research (NCBJ), Warsaw, Poland

30 Horia Hulubei National Institute of Physics and Nuclear Engineering, Bucharest-Magurele, Romania

31 Petersburg Nuclear Physics Institute (PNPI), Gatchina, Russia

32 Institute of Theoretical and Experimental Physics (ITEP), Moscow, Russia

33 Institute of Nuclear Physics, Moscow State University (SINP MSU), Moscow, Russia

34 Institute for Nuclear Research of the Russian Academy of Sciences (INR RAN), Moscow, Russia

35 Yandex School of Data Analysis, Moscow, Russia

36 Budker Institute of Nuclear Physics (SB RAS), Novosibirsk, Russia

37 Institute for High Energy Physics (IHEP), Protvino, Russia

38 ICCUB, Universitat de Barcelona, Barcelona, Spain

39 Universidad de Santiago de Compostela, Santiago de Compostela, Spain

40 European Organization for Nuclear Research (CERN), Geneva, Switzerland

41 Institute of Physics, Ecole Polytechnique Fédérale de Lausanne (EPFL), Lausanne, Switzerland

42 Physik-Institut, Universität Zürich, Zürich, Switzerland

43 Nikhef National Institute for Subatomic Physics, Amsterdam, The Netherlands

44 Nikhef National Institute for Subatomic Physics and VU University Amsterdam, Amsterdam, The Netherlands

45 NSC Kharkiv Institute of Physics and Technology (NSC KIPT), Kharkiv, Ukraine

46 Institute for Nuclear Research of the National Academy of Sciences (KINR), Kyiv, Ukraine

47 University of Birmingham, Birmingham, United Kingdom

48 H.H. Wills Physics Laboratory, University of Bristol, Bristol, United Kingdom

49 Cavendish Laboratory, University of Cambridge, Cambridge, United Kingdom

50 Department of Physics, University of Warwick, Coventry, United Kingdom

51 STFC Rutherford Appleton Laboratory, Didcot, United Kingdom

52 School of Physics and Astronomy, University of Edinburgh, Edinburgh, United Kingdom

53 School of Physics and Astronomy, University of Glasgow, Glasgow, United Kingdom

54 Oliver Lodge Laboratory, University of Liverpool, Liverpool, United Kingdom

55 Imperial College London, London, United Kingdom

56 School of Physics and Astronomy, University of Manchester, Manchester, United Kingdom

57 Department of Physics, University of Oxford, Oxford, United Kingdom

58 Massachusetts Institute of Technology, Cambridge, MA, United States

59 University of Cincinnati, Cincinnati, OH, United States

60 University of Maryland, College Park, MD, United States

61 Syracuse University, Syracuse, NY, United States

62 Pontifícia Universidade Católica do Rio de Janeiro (PUC-Rio), Rio de Janeiro, Brazil, associated to ${ }^{2}$

63 University of Chinese Academy of Sciences, Beijing, China, associated to ${ }^{3}$

64 School of Physics and Technology, Wuhan University, Wuhan, China, associated to ${ }^{3}$

65 Institute of Particle Physics, Central China Normal University, Wuhan, Hubei, China, associated to ${ }^{3}$ 
66 Departamento de Fisica, Universidad Nacional de Colombia, Bogota, Colombia, associated to ${ }^{8}$

67 Institut für Physik, Universität Rostock, Rostock, Germany, associated to ${ }^{12}$

68 National Research Centre Kurchatov Institute, Moscow, Russia, associated to ${ }^{32}$

69 Instituto de Fisica Corpuscular, Centro Mixto Universidad de Valencia-CSIC, Valencia, Spain, associated to ${ }^{38}$

70 Van Swinderen Institute, University of Groningen, Groningen, The Netherlands, associated to ${ }^{43}$

a Universidade Federal do Triângulo Mineiro (UFTM), Uberaba-MG, Brazil

${ }^{b}$ Laboratoire Leprince-Ringuet, Palaiseau, France

c P.N. Lebedev Physical Institute, Russian Academy of Science (LPI RAS), Moscow, Russia

d Università di Bari, Bari, Italy

e Università di Bologna, Bologna, Italy

${ }^{f}$ Università di Cagliari, Cagliari, Italy

$g$ Università di Ferrara, Ferrara, Italy

$h$ Università di Genova, Genova, Italy

i Università di Milano Bicocca, Milano, Italy

j Università di Roma Tor Vergata, Roma, Italy

${ }^{k}$ Università di Roma La Sapienza, Roma, Italy

${ }^{l}$ AGH - University of Science and Technology, Faculty of Computer Science, Electronics and Telecommunications, Kraków, Poland

$m$ LIFAELS, La Salle, Universitat Ramon Llull, Barcelona, Spain

${ }^{n}$ Hanoi University of Science, Hanoi, Viet Nam

- Università di Padova, Padova, Italy

$p$ Università di Pisa, Pisa, Italy

$q$ Università degli Studi di Milano, Milano, Italy

$r$ Università di Urbino, Urbino, Italy

$s$ Università della Basilicata, Potenza, Italy

${ }^{t}$ Scuola Normale Superiore, Pisa, Italy

u Università di Modena e Reggio Emilia, Modena, Italy

$v$ Iligan Institute of Technology (IIT), Iligan, Philippines

w Novosibirsk State University, Novosibirsk, Russia

$\dagger$ Deceased 